\title{
TORIC AND TROPICAL COMPACTIFICATIONS OF HYPERPLANE COMPLEMENTS
}

\author{
GRAHAM DENHAM
}

\begin{abstract}
These lecture notes survey and compare various compactifications of complex hyperplane arrangement complements. In particular, we review the Gel'fand-MacPherson construction, Kapranov's visible contours compactification, and De Concini and Procesi's wonderful compactification. We explain how these constructions are unified by some ideas from the modern origins of tropical geometry.
\end{abstract}

\section{CONTEnTs}

1. Introduction

2. Hyperplane Arrangements

3. Toric varieties

4. Tropical aspects

5. Compactifications

6. Concluding remarks

\section{Introduction}

The purpose of this paper is to give a gentle introduction to toric and tropical compactifications of linear spaces. The material is based on lectures given at the graduate student summer school "Arrangements in Pyrénées" in June 2012. The presentation gives emphasis to explicit examples and calculations, and we suggest some exercises for the reader. The material here can be found in various sources, and we give a guide to the literature without attempting to be comprehensive. Some proofs are included, particularly when it is possible to make a short or self-contained argument.

We review some terminology of matroid theory in $\S 2$, and note the relationship between matroid realizations, hyperplane arrangements, and linear subspaces of algebraic tori. In $\S 3$, we briefly recall those aspects of the theory of toric varieties that play a key role in the constructions we consider. After some discussion of some basics of tropical geometry ( $\$ 4$ ), arrangement compactifications appear in $\S 5$. We single out two important developments in the field. The first is Gel'fand, Goresky, MacPherson and Serganova's construction of the matroid stratification of the Grassmannian, [GGMS87, GM82]. The second is De Concini and

Date: June 18, 2013.

2000 Mathematics Subject Classification. Primary 52C35, Secondary 05B35 .

Key words and phrases. hyperplane arrangement, Bergman fan, tropicalization, wonderful compactification. Partially supported by a grant from NSERC of Canada. 
Procesi's wonderful compactification [DCP95]. We outline in detail some discoveries that tie the two together, due to Feichtner and Sturmfels [FS05], and Ardila and Klivans [AK06].

\section{Hyperplane Arrangements}

2.1. Matroids. A matroid is a structure that abstracts the linear (in)dependence properties of a finite collection of vectors. Matroids have numerous equivalent definitions, and we mention the book [Oxl11] as a detailed reference. As a brief introduction, let us define a matroid $M$ to be a nonempty collection of subsets $\mathcal{B}$ of a finite set $E$ that possesses the following property. That is, if $B, B^{\prime} \in \mathcal{B}$, and $x \in B-B^{\prime}$, then there is an element $y \in B^{\prime}-B$ for which $(B-\{x\}) \cup\{y\} \in \mathcal{B}$.

Then $\mathcal{B}$ is called the set of bases of the matroid. One can prove that all bases have the same cardinality. This number is called the rank of the matroid, an integer $d \geq 0$. Let

$$
\operatorname{In}(\mathrm{M})=\{I \subseteq E: I \subseteq B \text { for some } B \in \mathcal{B}\},
$$

called the independent sets of $\mathrm{M}$. The reader may notice that this exactly to say that the $\operatorname{In}(\mathrm{M})$ forms a simplicial complex with vertices $E$ in which the bases are the maximal simplices. This is called the matroid complex of $\mathrm{M}$.

Without loss, we will assume $E=[n]:=\{1,2, \ldots, n\}$, for some $n \geq 1$. The rank of a set $S \subseteq[n]$ is defined to be the cardinality of its largest independent subset, which we denote by $r(S)$. We define the closure of a set $S$ to be

$$
\operatorname{cl}(S)=\bigcup_{\begin{array}{c}
T: S \subseteq T \subseteq[n], \\
r(T)=r(S)
\end{array}} T .
$$

A set $X \subseteq[n]$ for which $\operatorname{cl}(X)=X$ is called a flat of $\mathrm{M}$. Let $L(\mathrm{M})$ denote the set of flats $\mathrm{M}$, and $L_{p}(\mathrm{M})$ the subset of flats of rank $p$, for $0 \leq p \leq d$. The set $L(\mathrm{M})$ is partially ordered by inclusion. In fact, $L(\mathrm{M})$ is a lattice, with operations

$$
\begin{aligned}
& X \wedge Y=X \cap Y \text { and } \\
& X \vee Y=\operatorname{cl}(X \cup Y) .
\end{aligned}
$$

It turns out that the lattice $L(\mathrm{M})$ is geometric, and all geometric lattices arise in this way (for which, see [Oxl11, §1.7]). $L(\mathrm{M})$ has a minimal element, $\hat{0}:=\operatorname{cl}(\emptyset)$.

If $\mathrm{M}$ is a matroid on $E$ with bases $\mathcal{B}$, the dual matroid $\mathrm{M}^{*}$ is the matroid with bases $\{E-B: B \in \mathcal{B}\}$. In particular, $\operatorname{rank}(\mathrm{M})+\operatorname{rank}\left(\mathrm{M}^{*}\right)=n$. Clearly the construction of duals is an involution, and it abstracts the idea of orthogonal complements: see Example 2.10 below.

Finally, an element $x \in[n]$ for which $r(\{x\})=0$ is a loop, and distinct elements $x, y \in[n]$ are parallel if $r(\{x, y\})=1$. A matroid $\mathrm{M}$ is simple if it has no loops or parallel edges.

2.2. Restrictions and sums. If $X \subseteq[n]$, then the set $\{I \cap X: I \in \operatorname{In}(\mathrm{M})\}$ defines the independent sets of a matroid on $X$. This is denoted $\mathrm{M} \mid X$, the restriction of $\mathrm{M}$ to $X$. If $X$ is a flat of $\mathrm{M}$, then flats of $\mathrm{M} \mid X$ are exactly those flats of $\mathrm{M}$ contained in $X$, which is to say that $L(\mathrm{M} \mid X)=[\hat{0}, X]$, an interval in $L(\mathrm{M})$.

Dually, for $X \subseteq[n]$, the contraction of $\mathrm{M}$ by $X$, written $\mathrm{M} / X$, is the matroid on the set $[n]-X$ defined by the rank function

$$
r_{\mathrm{M} / X}(S):=r_{\mathrm{M}}(S \cup X)-r_{\mathrm{M}}(X),
$$

for $S \subseteq[n]-X$. The flats of $\mathrm{M} / X$ are in bijection with flats of M containing $X$, so $L(\mathrm{M} / X) \cong$ $[\operatorname{cl}(X),[n]]:$ see [Oxl11, Prop. 3.3.8]. If $X$ is not a flat of $\mathrm{M}$, then any element $e \in \operatorname{cl}(X)-X$ is a loop in $\mathrm{M} / X$. 
If $\mathrm{M}_{1}$ and $\mathrm{M}_{2}$ are matroids on $E_{1}$ and $E_{2}$, respectively, the sum $\mathrm{M}=\mathrm{M}_{1} \oplus \mathrm{M}_{2}$ is defined to have bases

$$
\mathcal{B}=\left\{B_{1} \cup B_{2}: B_{1} \in \mathcal{B}\left(\mathrm{M}_{1}\right), B_{2} \in \mathcal{B}\left(\mathrm{M}_{2}\right)\right\} .
$$

Alternatively, the independence complex of $\mathrm{M}$ is the simplicial join $\operatorname{In}\left(M_{1}\right) * \operatorname{In}\left(M_{2}\right)$, and $L(\mathrm{M})=L\left(\mathrm{M}_{1}\right) \times L\left(\mathrm{M}_{2}\right)$. A matroid is connected if it cannot be (nontrivially) decomposed as a sum of matroids. Both $E_{1}$ and $E_{2}$ are flats of $\mathrm{M}_{1} \oplus \mathrm{M}_{2}$; conversely, $\mathrm{M}$ is connected provided that there are no sets $X \subseteq[n]$ for which $X$ and $[n]-X$ are both flats of M. The obvious notion of connected components is well-defined, and we denote the number of them by $\kappa(\mathrm{M})$. A flat $X$ is said to be irreducible if the restriction $\mathrm{M} \mid X$ is connected. We let $L_{\text {irr }}(\mathrm{M})$ denote the set of irreducible flats.

2.3. Polytopes. There is a remarkable relationship between matroids, convex geometry and toric varieties, discovered by Gel'fand, Goresky, MacPherson, and Serganova [GGMS87]. In order to begin to describe it, let $e_{1}, \ldots, e_{n}$ be the standard basis for $\mathbb{Z}^{n}$, and for any subset $S \subseteq[n]$, let $e_{S}=\sum_{i \in S} e_{i}$.

If $\mathrm{M}$ is a matroid on $E=[n]$, let

$$
P_{\mathrm{M}}=\operatorname{conv}\left\{e_{B}: B \in \mathcal{B}\right\} \subseteq \mathbb{R}^{n},
$$

the convex hull of the indicator vectors on the bases of $\mathrm{M}$. This is the matroid polytope of $\mathrm{M}$. If $\mathrm{M}$ has rank $d$, then $P_{\mathrm{M}} \subseteq d \cdot \Delta^{n-1}$, where $\Delta^{n-1}=\operatorname{conv}\left(\left\{e_{1}, \ldots, e_{n}\right\}\right) \subseteq \mathbb{R}^{n}$, the standard $(n-1)$-dimensional simplex.

If $\mathrm{M}$ is connected, then $P_{\mathrm{M}}$ has dimension $n-1$. If $\mathrm{M}$ decomposes as a sum, then $P_{\mathrm{M}}$ is a corresponding cartesian product, so the dimension of $P_{\mathrm{M}}$ is $n-\kappa(\mathrm{M})$ (see [FS05]). Faces of the matroid polytope are themselves matroid polytopes. Such matroids are obtained from $M$ by deleting independent sets, so are called degenerations of $\mathrm{M}$. If $F$ is a face of $P_{\mathrm{M}}$, let $\mathrm{M}_{F}$ denote the matroid whose bases are the vertices of the face $F$. For a linear functional $u \in \mathbb{R}^{n}$, let $F$ be the face of $P_{\mathrm{M}}$ on which $u$ achieves its minimum, and let $\mathrm{M}_{u}$ denote the matroid corresponding to the face: then $F=P_{\mathrm{M}_{u}}$.

The face structure of the matroid polytope was worked out as follows in [AK06, FS05]. We assume that $\mathrm{M}$ is connected. For $u \in \mathbb{R}^{n}$, let

$$
\mathcal{F}(u):=\left(\emptyset=F_{0} \subset F_{1} \subset \cdots \subset F_{k}=[n]\right)
$$

be the (unique) chain of subsets for which the coefficients $\left\{u_{i}: i \in F_{a}-F_{a-1}\right\}$ are constant for all $1 \leq a \leq k$, and $u_{i} \leq u_{j}$ whenever $i \in F_{a}$ and $j \in F_{b}$ for $a<b$.

Then the face of $P_{\mathrm{M}}$ that minimizes $u$ depends only on $\mathcal{F}(u)$, and

$$
\mathrm{M}_{u}=\bigoplus_{i=1}^{k}\left(M \mid F_{i}\right) / F_{i-1} .
$$

Theorem 2.1 ([AK06, FS05]). The following are equivalent for a vector $u \in \mathbb{R}^{n}$ :

- The matroid $\mathrm{M}_{u}$ has no loops;

- The subsets in the chain $\mathcal{F}(u)$ are flats of $\mathrm{M}$;

- The face $F=P_{\mathrm{M}_{u}}$ is not contained in the boundary of the simplex $\partial\left(d \cdot \Delta^{n-1}\right)$.

If $i$ is a loop in $\mathrm{M}_{u}$, then $e_{i}$ is an (inner) normal vector for $F$.

Clearly the number of components $\kappa\left(\mathrm{M}_{u}\right) \geq k$; if in fact this is an equality, then $P_{\mathrm{M}_{u}}$ is face of codimension $k-1$ in $P_{\mathrm{M}}$. In the case where $k=2$, we obtain a description of facets. That is, if $\mathrm{M}_{u}$ contains no loop, then $\mathcal{F}(u)=(\emptyset \subset X \subset[n])$ for some proper flat $X$. We may shift $u$ by a multiple of the vector $e_{[n]}$ and rescale by a positive number without changing the face $P_{\mathrm{M}_{u}}$, so we may assume that $u=-e_{X}$. 


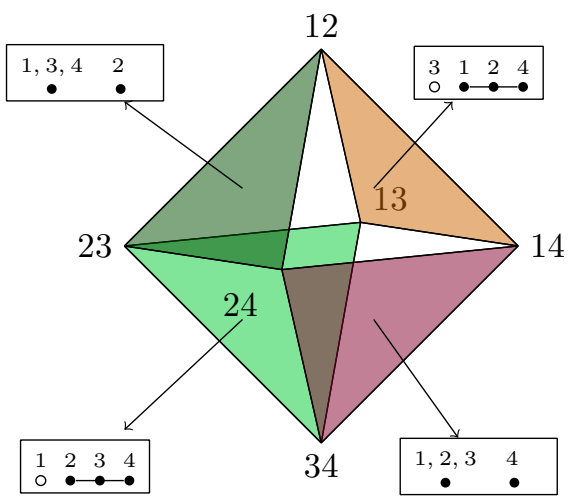

(a) $P_{\mathrm{M}}$ and some degenerations

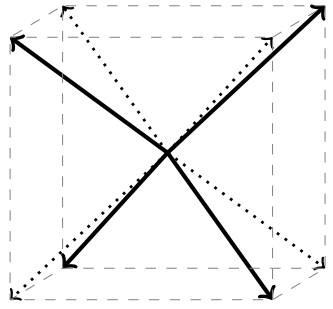

(b) Rays of the fan $\Sigma_{M}$

FigURE 1. The matroid polytope of $U_{2,4}$

Theorem $2.2([\mathrm{FS} 05])$. A facet $F$ of $P_{\mathrm{M}}$ which is not contained in $\partial\left(d \cdot \Delta^{n-1}\right)$ has an inner normal vector $-e_{X}$ for some flat $X \in L(\mathcal{A})$. Such facets are in bijection with the set

$$
\{X \in L(\mathcal{A}): \mathrm{M} \mid X \text { and } \mathrm{M} / X \text { are both connected. }\}
$$

For reasons which will be more evident later, it will be convenient to think of outer normal vectors of matroid polytopes, instead of inner ones.

Definition 2.3. For a matroid $M$, let $\Sigma_{M}$ denote the outer normal fan of the matroid polytope $P_{\mathrm{M}}$. By definition, this is an equivalence relation on vectors in $\mathbb{R}^{n} / \mathbb{R}(1,1, \ldots, 1)$, where $u \sim v$ if and only if $u$ and $v$ achieve their maximum value on the same face of $P_{\mathrm{M}}$. The equivalence classes form the relative interiors of polyhedral cones: we refer to [Zie95, §7.1] for background.

Example 2.4. For integers $1 \leq d \leq n$, the uniform matroid $U_{d, n}$ has underlying set $[n]$, and its bases consist of all subsets of $[n]$ of size $d$. The matroid polytope of $U_{2,4}$ is a 3 -dimensional polytope in $\mathbb{R}^{4}$. It is shown in Figure 1 with the facets of the form (2.2) shaded.

Remark 2.5. Various other polytopes can be associated with a matroid, and the reader should see [Sch03, Ch. 40] for details. In particular, the independent set polytope is the convex hull of indicator functions of all independent sets, and $P_{\mathrm{M}}$ is its facet with normal vector $e_{[n]}$. $\diamond$

Exercise 2.6. Show that if $M$ is a matroid on the set $[n]$, then the matroid polytopes for $M$ and its dual are related by $P_{\mathrm{M}^{*}}=e_{[n]}-P_{\mathrm{M}}$, where "-" denotes reflection through the origin. Conclude the outer normal fan of $P_{\mathrm{M}}$ is the inner normal fan of $P_{\mathrm{M}^{*}}$.

2.4. Linear matroids. A hyperplane arrangement is, informally, a linear realization of a matroid with no loops. (Our standard reference for hyperplane arrangements is the book [OT92].) In order to make this informal notion precise, let $\mathbb{k}$ be an algebraically closed field, and let $V$ be a $d$-dimensional linear subspace of $\mathbb{k}^{n}$, where $d \geq 1$ If we let $f: V \hookrightarrow \mathbb{k}^{n}$ denote the inclusion, its $i$ th coordinate is a linear map, $f_{i} \in V^{*}$. $V$ determines a matroid $\mathrm{M}(V)$ on the set of coordinates $[n]$ : one declares a set $I \subseteq[n]$ to be an independent set of $\mathrm{M}(V)$ if and only if the set $\left\{f_{i}: i \in I\right\}$ is linearly independent. Then the remaining matroid-theoretic terms have straightforward linear-algebraic counterparts. Such a matroid is said to be linear, or a linear realization of its isomorphism type. 
Let $x_{1}, \ldots, x_{n}$ be coordinate functions on $\mathbb{k}^{n}$, and let $\mathcal{C}_{n}=\left\{\hat{H}_{1}, \ldots, \hat{H}_{n}\right\}$ denote the set of coordinate hyperplanes, $\hat{H}_{i}=\operatorname{ker} x_{i}$ for $1 \leq i \leq n$. Note that $V \subseteq \hat{H}_{i}$ if and only if $f_{i}=0$, which is equivalent to $i$ being a loop of $\mathrm{M}(V)$. Provided that $\mathrm{M}(V)$ has no loops, $H_{i}:=\hat{H}_{i} \cap V$ is a hyperplane of $V$ for all $i$. Let $\mathcal{A}=\mathcal{A}(V)=\left\{H_{1}, \ldots, H_{n}\right\}$. This is a (central, essential) hyperplane arrangement in $V$. By construction, $H_{i}=\operatorname{ker} f_{i}$ for $1 \leq i \leq n$, so $\{i, j\}$ is dependent in $\mathrm{M}(V)$ if and only if $H_{i}=H_{j}$. Thus a simple matroid $\mathrm{M}(V)$ gives an arrangement of distinct hyperplanes. Conversely, a set of $n$ hyperplanes in a $d$-dimensional linear space $V$ is called essential if $\bigcap_{i=1}^{n} H_{i}=\{0\}$, and in this case, any choice of defining equations for the hyperplanes give a linear realization of a simple matroid with no loops.

Example 2.7. Choose integers $1 \leq d \leq n$, and let $A=A_{d, n}$ be a $d \times n$ matrix for which any $d$ columns are linearly independent. Let $V=\operatorname{row}(A)$, the span of the rows. The matroid $\mathrm{M}(V)$ is a realization of the uniform matroid $U_{d, n}$. The independent sets consist of all subsets of $[n]$ of cardinality at most $d$. The hyperplane arrangement $\mathcal{A}$ is called the generic arrangement of $n$ hyperplanes in $\mathbb{k}^{n}$. If $n=d$, then $V=\mathbb{k}^{n}$, and $\mathcal{A}=\mathcal{C}_{n}$, called the Boolean arrangement of rank $n$. If $d=2, \mathcal{A}$ consists of $n$ lines in the plane through the origin.

Example 2.8. Let $V=\operatorname{row}(A)$, where

$$
A=\left(\begin{array}{ccccc}
1 & 0 & 0 & 1 & 1 \\
0 & 1 & 0 & -1 & 0 \\
0 & 0 & 1 & 0 & -1
\end{array}\right) ; \quad \mathrm{M}(V):
$$

where the matroid diagram encodes the flats as collinear subsets, and the arrangement $\mathcal{A}(V)$ is drawn in a suitable affine chart of $\mathbb{P}^{2}$. Here, $n=5, d=3$, and the linear functionals are $\{u, v, w, u-v, u-w\}$.

Example 2.9. For $d \geq 2$, let $V=V\left(A_{d}\right)=\mathbb{C}^{d+1} / \mathbb{C} e_{[d+1]}$, where $e_{[d+1]}=(1,1, \ldots, 1)$, and let $f_{i j}=z_{i}-z_{j}$ for $1 \leq i<j \leq d+1$. Then $f: V \rightarrow \mathbb{C}^{n}$ is a linear inclusion, where $n=\left(\begin{array}{l}d \\ 2\end{array}\right)$, and $\mathrm{M}(V)$ is the matroid of the complete graph, $K_{d+1}$. Moreover,

$$
U(\mathcal{A})=\left\{z \in V: z_{i} \neq z_{j} \text { for } i \neq j\right\}
$$

is the complement of the reflecting hyperplanes of the $A_{d}$ root system, also known as the (complex) braid arrangement. One also writes $U(\mathcal{A})$ as $F(\mathbb{C}, d+1)$, the configuration space of $d+1$ (distinct, labelled) points in $\mathbb{C}$.

The flats $X$ of $\mathcal{A}$ are indexed by partitions $\pi_{X}$ of the set $[d+1]$, by putting indices $i$ and $j$ in the same block of $\pi_{X}$ if and only if $z_{i}=z_{j}$ on $X$. Then $X \leq Y$ if and only if $\pi_{X}$ refines $\pi_{Y}$, and the rank of $X$ equals $d$ minus the number of blocks in $\pi_{X}$. A flat is irreducible if and only if $\pi_{X}$ has exactly one block of size greater than one.

Example 2.10. If $\mathrm{M}=\mathrm{M}(V)$ is linear, then the dual matroid $\mathrm{M}^{*}=\mathrm{M}\left(V^{\perp}\right)$, where $V^{\perp}$ denotes the orthogonal complement of $V$ in the dual vector space $\left(\mathbb{k}^{n}\right)^{*}$.

Remark 2.11. It can be useful to think of $V$ as a point in the Grassmannian $\operatorname{Gr}_{d, n}$, which embeds in $\mathbb{P}\left(\bigwedge^{d} \mathbb{k}^{n}\right)$ via the Plücker embedding. Homogeneous coordinates for the latter space are indexed by subsets $I \subseteq[n]$ of size $d$ : let $x_{I}=x_{i_{1}} \wedge \cdots \wedge x_{i_{d}}$, where $I=\left\{i_{1}, \ldots, i_{d}\right\}$ and $i_{1}<\ldots<i_{d}$. Translating our remarks above into this notation, we see the $I$ th coordinate of $V$ is nonzero if and only if $I$ is independent in $\mathrm{M}(V)$. 
2.5. Linear restrictions and sums. A linear matroid decomposes as a matroid sum if and only if the vector space $V$ has a splitting which is compatible with the coordinates in $\mathbb{k}^{n}$. More precisely, for any subset $S \subseteq[n]$, let

$$
\mathbb{k}^{S}=\left\{x \in \mathbb{k}^{n}: x_{i}=0 \text { for } i \notin S\right\}
$$

denote the coordinate subspace. If the matroid $\mathrm{M}(V)=\mathrm{M}_{1} \oplus \mathrm{M}_{2}$ where $\mathrm{M}_{i}$ is a matroid on $E_{i}$, for $i=1,2$, it is not hard to check that $\mathrm{M}_{i}=\mathrm{M}\left(V_{i}\right)$, where $V \cong V_{1} \oplus V_{2}$, and $V_{i}=V \cap \mathbb{k}^{E_{i}}$. Conversely, suppose $V_{i} \subseteq \mathbb{k}^{E_{i}}$ for $i=1,2$, where $[n]=E_{1} \cup E_{2}$ is a partition with nonempty parts. Let $V=V_{1} \oplus V_{2}$ : then $\mathrm{M}(V)=\mathrm{M}\left(V_{1}\right) \oplus \mathrm{M}\left(V_{2}\right)$.

Along the same lines, if $X \subseteq[n]$ is a flat of $\mathrm{M}=\mathrm{M}(V)$, let $V_{X}$ denote the image of $V$ under the coordinate projection map $\mathbb{k}^{n} \rightarrow \mathbb{k}^{X}$. Then $V_{X}$ is a linear quotient of $V$, and a realization of $\mathrm{M} \mid X$ in $\mathbb{k}^{X}$.

2.6. A torus action. It should be clear that multiplying each coordinate map $f_{i}$ by a nonzero scalar does not change the matroid or set of hyperplanes $\mathcal{A}$. That is, let $T^{n}=\left(\mathbb{k}^{\times}\right)^{n}$ denote the algebraic torus of rank $n$, which acts on $\mathbb{k}^{n}$ by (left) multiplication: then $\mathrm{M}(t \cdot V)=\mathrm{M}(V)$ for all $t \in T^{n}$.

Of course, if the coordinates of $t \in T^{n}$ are all equal, $t$ acts on $\mathbb{k}^{n}$ by scalar multiplication, and $t \cdot V=V$. So we should instead consider the quotient torus $T_{0}^{n-1}:=T^{n} / \mathbb{k}^{\times}$by the diagonal one-parameter subgroup. In other words, $T^{n}$ acts on the Grassmannian $\mathrm{Gr}_{d, n}$ via its action on $\mathbb{k}^{n}$, and the action factors through $T_{0}^{n-1}$.

In this language, the matroid structure is constant on each torus orbit $T_{0}^{n-1} \cdot V \subseteq \mathrm{Gr}_{d, n}$. The hyperplane arrangements $\mathcal{A}(V)$ at different points in the orbit have different ambient spaces, but they are linearly isomorphic. It remains to consider stabilizers of the torus action on $\mathrm{Gr}_{d, n}$. It turns out that $T_{0}^{n-1}$ acts freely on $V$ when $\mathrm{M}(V)$ is connected:

Proposition 2.12. If $\mathrm{M}(V)$ has $\kappa$ connected components, then

$$
\operatorname{stab}_{T^{n}}(V) \cong\left(\mathbb{k}^{\times}\right)^{\kappa} .
$$

Proof. Suppose that $[n]=E_{1} \dot{\cup} \cdots \dot{\cup} E_{\kappa}$ is the decomposition into connected components. We claim

$$
\operatorname{stab}_{T^{n}}(V)=\left\{t \in T^{n}: t_{i}=t_{j} \text { provided } i, j \in E_{k} \text { for some } k\right\} .
$$

Since $V=V_{1} \oplus \cdots \oplus V_{\kappa}$ where $V_{i}=V \cap \mathbb{k}^{E_{i}}$ for $1 \leq i \leq \kappa$, scalar multiplication on each factor shows that the right-hand side of (2.3) is included in the left. To show the other inclusion, suppose $t \in \operatorname{stab}_{T^{n}}(V)$. The eigenspaces of $t$ as an endomorphism of $\mathbb{k}^{n}$ are simply coordinate subspaces, indexed by the partition of $[n]$ into subsets $F_{1}, \ldots, F_{l}$ on which the coordinates of $t$ are constant (and pairwise distinct). By hypothesis, the action of $t$ restricts to $V$, so

$$
V=\left(V \cap \mathbb{k}^{F_{1}}\right) \oplus \cdots \oplus\left(V \cap \mathbb{k}^{F_{l}}\right) .
$$

It follows that the partition $E_{1}, \ldots, E_{\kappa}$ refines this one, and $t$ is contained in the right-hand side of (2.3).

In particular, if $\mathrm{M}(V)$ is connected, then the orbit $T^{n} \cdot V \cong T_{0}^{n-1}$. This provides an example of a torus torsor in the Grassmannian. 
2.7. Arrangement complements. For any hyperplane arrangement $\mathcal{A}$ in $V$, let $U(\mathcal{A})=$ $V-\bigcup_{i} H_{i}$. This is both the complement of the hypersurface $f^{-1}(0)$ as well as an irreducible, closed subvariety of the torus $T^{n}$, since $U(\mathcal{A})=V \cap T^{n}$. It is useful to keep in mind both points of view. The torus is, itself, the complement of the Boolean arrangement $\mathcal{C}^{n}$. The space $U(\mathcal{A})$ is a central object of study in the theory of hyperplane arrangements, particularly when $\mathbb{k}=\mathbb{C}$ : we refer to the forthcoming book $\left[\mathrm{CDF}^{+}\right]$.

Let $\mathbb{P} U(\mathcal{A}):=U(\mathcal{A}) / \mathbb{k}^{\times}$, where $\mathbb{k}^{\times}$is the diagonal subgroup again. This is a subvariety of the projective space $\mathbb{P} V$ : as above, $\mathbb{P} U(\mathcal{A})=\mathbb{P} V \cap T_{0}^{n-1}$. The quotient torus splits as a coordinate subtorus of $T^{n}$. For $1 \leq i \leq n$, let $T_{i}^{n-1}=\left\{t \in T: t_{i}=1\right\}$, and consider the isomorphism of groups $c_{i}: T_{0}^{n-1} \rightarrow T_{i}^{n-1}$, given by $c_{i}(t)=t_{i}^{-1} \cdot t$. By restricting $c_{i}$ to $\mathbb{P} U(\mathcal{A})$, we see that $\mathbb{P} U(\mathcal{A})$ is isomorphic to the subvariety of $U(\mathcal{A})$ on which $f_{i}=1$.

Exercise 2.13. Check that $U(\mathcal{A}) \cong \mathbb{P} U(\mathcal{A}) \times \mathbb{k}^{\times}$.

Remark 2.14. An irreducible, closed subvariety of an algebraic torus is said to be very affine. Another very affine variety associated with a hyperplane arrangement is given as follows. Given a lattice vector $m \in \mathbb{Z}^{n}$ with $\operatorname{gcd}\left\{m_{i}: i \in[n]\right\}=1$, form the subgroup

$$
T^{m}=\left\{t \in T: t_{1}^{m_{1}} t_{2}^{m_{2}} \cdots t_{n}^{m_{n}}=1\right\},
$$

and let $F(\mathcal{A}, m)=V \cap T^{m}$. In general, this is a level set of a master function

$$
\prod_{i=1}^{n} f_{i}^{m_{i}}: U(\mathcal{A}) \rightarrow \mathbb{k}^{\times}
$$

See, for example, [Var11], for more details. If $m_{i}>0$ for each $1 \leq i \leq n$, the variety $F(\mathcal{A}, \mathbf{m})$ is an (unreduced) Milnor fibre of $\mathcal{A}$ with given multiplicities. If each $m_{i}=1$, it coincides with the usual, global Milnor fibre of the hypersurface. These varieties are qualitatively quite different from the hyperplane complements, and their topology is more subtle: see the paper by Suciu in this volume [Suc13]. It would be interesting to know if there is anything special about the topology of intersections of linear spaces $V \subseteq \mathbb{k}^{n}$ and general subtori of $T^{n}$.

\section{TORIC VARIETIES}

For background on this subject, we recommend the excellent book by Cox, Little and Schenck [CLS11]. In order to keep these notes self-contained, we will give a brief outline of the role of polyhedral combinatorics in the theory of toric varieties. From now on, we will assume that $\mathbb{k}=\mathbb{C}$.

We recall that a (normal) toric variety of dimension $n$ to be an irreducible, normal complex variety $X$ for which

- $X$ contains a dense, open subvariety isomorphic to a complex torus $T^{n}$;

- the action of $T^{n}$ on itself extends to $X$, giving an algebraic map $T^{n} \times X \rightarrow X$.

3.1. Cones and orbits. One foundational part of the theory is that toric varieties are stratified by closures of torus orbits, and that this stratification determines the toric variety's isomorphism type. The orbits are described combinatorially by means of a polyhedral fan, denoted $\Sigma_{X}$, and we recall briefly the nature of this description.

The one-parameter subgroups of $T^{n}$ form an integer lattice, $N:=\operatorname{Hom}\left(\mathbb{C}^{\times}, T^{n}\right)$, which is isomorphic to $\mathbb{Z}^{n}$. Suppose a toric variety $X$ containing $T^{n}$ is given. Then, for each $u \in N$, we check whether or not the homomorphism $u$ : $\mathbb{C}^{\times} \rightarrow T^{n} \subseteq X$ can be extended continuously to a map $u: \mathbb{C} \rightarrow X$. That is, let

$$
\left|\Sigma_{X, \mathbb{Z}}\right|=\left\{u \in N: \lim _{t \rightarrow 0} u(t) \text { exists. }\right\}
$$


Impose an equivalence relation by letting, for $u, v \in\left|\Sigma_{X, \mathbb{Z}}\right|$,

$$
u \sim v \text { if and only if } \lim _{t \rightarrow 0} u(t)=\lim _{t \rightarrow 0} v(t) .
$$

If $S \subseteq N$ is an equivalence class, let $N_{\mathbb{R}}=N \otimes_{\mathbb{Z}} \mathbb{R}$, a real Euclidean space, and define $\sigma \subseteq N_{\mathbb{R}}$ by

$$
\sigma=\overline{\mathbb{R}_{\geq 0} S},
$$

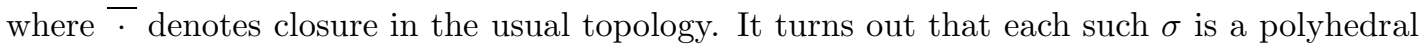
cone.

Let $\Sigma_{X}$ denote the set of all cones, which is (by construction) in bijection with the set of limit points of one-parameter subgroups. The set of cones $\Sigma_{X}$ is closed under intersection. For each $\sigma \in \Sigma_{X}$, let $O(\sigma)=T^{n} \cdot \lim _{t \rightarrow 0} u(t)$ denote the torus orbit coming from any $u \in\left|\Sigma_{X, \mathbb{Z}}\right| \cap \sigma$. All orbits arise this way, and the cones keep track of their incidence data: that is,

$$
O(\sigma) \subseteq \overline{O(\tau)} \text { if and only if } \tau \subseteq \sigma .
$$

Example 3.1. Affine space $\mathbb{C}^{n}$ is a toric variety whose fan $\Sigma_{\mathbb{C}^{n}}$ consists of cones $\sigma_{S}$, for all $S \subseteq[n]$, where $\sigma_{S}=\mathbb{R}_{\geq 0}\left\{e_{i}: i \in S\right\}$, and $\left\{e_{1}, \ldots, e_{n}\right\}$ are the standard basis for $N=\mathbb{Z}^{n}$. The cone $\sigma_{\emptyset}=\{0\} \subseteq N$ corresponds to the maximal torus orbit $T^{n} \subseteq \mathbb{C}^{n}$ in the dictionary above, while the full orthant $\sigma_{[n]}$ corresponds to the 0-dimensional orbit $\{0\} \subseteq \mathbb{C}^{n}$.

Example 3.2. The usual embedding $T_{0}^{n-1} \subseteq \mathbb{P}^{n-1}$ makes projective space a toric variety with $N=\mathbb{Z}^{n} / \mathbb{Z} e_{[n]}$. The toric fan $\Sigma_{\mathbb{P}^{n-1}}$ consists of cones $\sigma_{S} \subseteq \mathbb{R}^{n} / \mathbb{R} e_{[n]}$, for all $S \subsetneq[n]$, where $\sigma_{S}$ is defined as in Example 3.1. In particular, the one-dimensional cones $\sigma_{\{i\}}$ correspond to orbits of points $\left[x_{1}: \cdots: x_{n}\right] \in \mathbb{P}^{n-1}$ with $x_{i}=0$, for $1 \leq i \leq n$.

A cone $\sigma$ of dimension $d$ is simplicial if it is spanned by $d$ rays. A necessary condition for a toric variety $X$ to be smooth is that each cone of the fan $\Sigma_{X}$ is simplicial. A sufficient condition for $X$ to be smooth is that $\Sigma_{X}$ is simplicial, and that each cone $\sigma$ is unimodular: that is, $\sigma$ is generated by lattice vectors that extend to a basis of the lattice $N$.

3.2. Projective toric varieties. An important family of toric varieties is constructed in the following way. Let $A$ be a $n \times r$ integer matrix, and consider the group homomorphism between tori, $p_{A}: T^{n} \rightarrow T^{r}$, given by

$$
p(t)=\left(\prod_{i=1}^{n} t_{i}^{A_{i 1}}, \prod_{i=1}^{n} t_{i}^{A_{i 2}}, \ldots, \prod_{i=1}^{n} t_{i}^{A_{i r}}\right) .
$$

Then $p_{A}$ is injective provided that $A$ is unimodular of full rank, and $p_{A}$ induces a map of quotient tori $\bar{p}_{A}: T_{0}^{n-1} \rightarrow T_{0}^{r-1}$ provided that the sum of the entries in any two columns of $A$ are equal. If both of these conditions are satisfied, we have an embedding

$$
\bar{p}_{A}: T_{0}^{n-1} \rightarrow T_{0}^{r-1} \subseteq \mathbb{P}^{r-1}:
$$

let $X_{A}$ denote the toric variety given by taking the closure of the image of $\bar{p}_{A}$.

If one translates each column of $A$ by a fixed lattice vector to obtain a matrix $A^{\prime}$, then $\bar{p}_{A}=\bar{p}_{A^{\prime}}$, so $X_{A}=X_{A^{\prime}}$. Let $P_{A}$ denote the convex hull of the columns of $A$, a polytope in $\mathbb{Z}^{n}$. Choose a translation of the columns of $A$ so that the column sums are zero, so that $P_{A} \subseteq M:=N^{*} \cong \mathbb{Z}^{n-1}$.

In fact, any toric variety $X$ which is equivariantly embedded in projective space can be written as $X=X_{A}$ for some $A$, so we will simply write $P_{X}$ for (the translation equivalence class of) the polytope $P_{A}$.

The toric fan of $X_{A}$ is easy to describe: provided that $X_{A}$ is normal, the fan is the (inner) normal fan of $P_{A}$. This is a complete fan: $\left|\Sigma_{X, \mathbb{Z}}\right|=N$, and for $u, v \in N$, we have $u \sim v$ if and 
only if $u$ and $v$, regarded as linear functionals on $M$, both achieve their minimum on the same subset of $P_{A}$.

With this in mind, recall the Gel'fand-MacPherson construction from $\$ 2.6$ that embedded a torus in $\mathrm{Gr}_{d, n}$. If a linear matroid $\mathrm{M}(V)$ is connected, then the map $a_{V}: T_{0}^{n-1} \rightarrow \mathrm{Gr}_{d, n}$ defined by $a_{V}(t)=t \cdot V$ is injective, by Proposition 2.12. So the closure of the image is a toric variety,

$$
X_{\mathrm{M}}:=\overline{a_{V}\left(T_{0}^{n-1}\right)} \subseteq \mathrm{Gr}_{d, n} .
$$

The Grassmannian is complete, so $X_{M}$ is too. In fact, using the Plücker embedding, we can identify $X_{\mathrm{M}}$ with the closure of the image $T_{0}^{n-1}$ in the projective space $\mathbb{P}\left(\bigwedge^{d} \mathbb{C}^{n}\right)$, as in Remark 2.11, so $X_{\mathrm{M}}$ is a projective toric variety. It has a particularly nice description:

Theorem 3.3 ([GGMS87]). The weight polytope of $X_{\mathrm{M}}$ is the matroid polytope, $P_{\mathrm{M}}$.

Proof. We assume for simplicity that $\mathrm{M}(V)$ is connected, the general case being similar. Consider the composition of maps $b_{V}$,

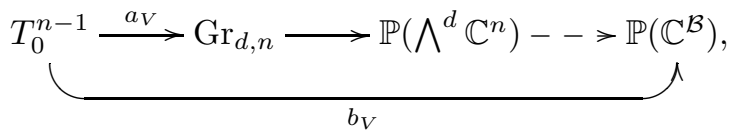

where last rational map is projection onto those $x_{I}$ for which $I$ is a base of $\mathrm{M}(V)$. Its restriction to the image of the torus is, in fact, an injective, equivariant regular map, and we want to calculate the weights of the embedding of $X_{\mathrm{M}}$ in $\mathbb{P}\left(\mathbb{C}^{\mathcal{B}}\right)$.

Suppose $V=\operatorname{row}(A)$ for some $d \times n$ matrix $A$. For $t \in T_{0}^{n-1}$, a basis for $t \cdot V$ is obtained by multiplying column $j$ of $A$ by $t_{j}$. For a set $I \subseteq[n]$ of size $d$, we write $\operatorname{det}\left(A_{I}\right)$ for the minor on columns $I$, which is also the $I$ th Plücker coordinate of $V$. Then for $I \in \mathcal{B}$, the columns of $A$ are independent, so $\operatorname{det}\left(A_{I}\right) \neq 0$, and the torus action on the $I$ th coordinate is given by

$$
b_{V}(t \cdot V)_{I}=\left(\prod_{j \in I} t_{j}\right) \cdot b_{V}(V)_{I}
$$

Reading the exponents of this monomial, we see that the weight vector for coordinate $I$ is the vector $e_{I}$, and these are the vertices of the matroid polytope.

By a result of White [Whi77], the toric variety $X_{\mathrm{M}}$ is normal. So the closures of torus orbits in $X_{\mathrm{M}}$ are in bijection with the faces of the matroid polytope $P_{\mathrm{M}}$. Since a face $F$ of $P_{\mathrm{M}}$ is the matroid polytope of a matroid $\mathrm{M}_{F}$, the closure of the corresponding orbit is another toric variety, $X_{\mathrm{M}_{F}}$. We illustrate with an example.

Example 3.4. Continuing Example 2.7, consider the arrangement of four lines in the plane, $\mathrm{M}(V) \cong U_{2,4}$. If we choose $f: \mathbb{C}^{2} \rightarrow \mathbb{C}^{4}$, to be $f=(z, w, z-w, z+w)$, then for $t \in T_{0}^{3}$, we have

$$
t \cdot V=\operatorname{row}\left(\begin{array}{cccc}
t_{1} & 0 & t_{3} & t_{4} \\
0 & t_{2} & -t_{3} & t_{4}
\end{array}\right)
$$

whose image in $\mathbb{P}^{5}$ is

$$
\left[t_{1} t_{2}:-t_{1} t_{3}:-t_{2} t_{3}: t_{1} t_{4}:-t_{2} t_{4}: 2 t_{3} t_{4}\right]
$$

Then the matrix of weights, with the columns ordered in this way, is

$$
A=\left(\begin{array}{llllll}
1 & 1 & 0 & 1 & 0 & 0 \\
1 & 0 & 1 & 0 & 1 & 0 \\
0 & 1 & 1 & 0 & 0 & 1 \\
0 & 0 & 0 & 1 & 1 & 1
\end{array}\right),
$$


and the convex hull of the columns is the octahedral matroid polytope of Figure 1. The normal fan of an octahedron is the fan in $N=\mathbb{Z}^{4} / \mathbb{Z} e_{[4]}$ obtained by taking cones over each face of the polar polytope, the cube. The fan is not simplicial, so the toric variety $X_{\mathrm{M}}$ is not smooth.

The coordinate $x_{1}$ takes its minimum on the facet $F=\operatorname{conv}\left\{e_{23}, e_{24}, e_{34}\right\}$, so the vector $u=(1,0,0,0) \in N$ spans a ray of the fan. We can work out the corresponding codimension-1 torus orbit by computing $\lim _{t \rightarrow 0} u(t)$, which is

$$
\lim _{t \rightarrow 0} \operatorname{row}\left(\begin{array}{cccc}
t^{1} & 0 & t^{0} & t^{0} \\
0 & t^{0} & -t^{0} & t^{0}
\end{array}\right)=\operatorname{row}\left(\begin{array}{cccc}
0 & 1 & -1 & 1 \\
0 & 0 & 1 & 1
\end{array}\right) .
$$

The matroid of this limit subspace has a loop, the element 1. By Theorem 2.1, the facet $F$ must lie in the boundary of the simplex $2 \cdot \Delta^{3}$, which it does. (See Figure 1 ).

Example 3.5. Recall the hyperplane arrangement from Example 2.8 with $d=3$ and $n=5$. Its matroid polytope $P_{\mathrm{M}}$ is 4-dimensional, and $|\mathcal{B}|=8$. Calculating by hand (or with help from Macaulay 2 [GS, Polyhedra package]), one finds $P_{\mathrm{M}}$ has 18 edges, 17 -faces, and 7 facets: see Figure 2(b). One facet $F$ is contained in $\partial\left(3 \cdot \Delta^{4}\right)$ and has vertices $\left\{e_{234}, e_{235}, e_{245}, e_{345}\right\}$. Again, 1 is a loop in $\mathrm{M}_{F}$, and $-e_{1}$ is an outer normal vector. Four facets, normal to $e_{i}$ for $i=2,3,4,5$ are square-based pyramids. The two facets normal to $e_{124}$ and $e_{135}$ are triangular prisms. The vector $e_{1}$ takes its maximum on their intersection, which is a square (2-dimensional) face, reflecting the fact that the contraction $\mathrm{M} /\{1\}$ is not connected: see Theorem 2.1.

We conclude the section with another example of how constructions in convex geometry are reflected by toric varieties. Let $X_{1}$ and $X_{2}$ be $n$-dimensional projective toric varieties, and $i_{j}: T^{n} \rightarrow X_{j}$ the inclusion of their maximal torus, for $j=1,2$. Consider the diagonal map $d: T^{n} \rightarrow X_{1} \times X_{2}$ given by $d(t)=\left(d_{1}(t), d_{2}(t)\right)$, and let $X=\overline{d\left(T^{n}\right)} \subseteq X_{1} \times X_{2}$.

Proposition 3.6 (Prop. 8.1.4, [GKZ94]). The space $X$ is also a projective toric variety, and $P_{X}=P_{X_{1}}+P_{X_{2}}$, where "+" denotes the Minkowski sum of polytopes.

Exercise 3.7. Define a map $T_{0}^{n} \rightarrow \mathbb{P}^{n} \times \mathbb{P}^{n}$ by $t \mapsto\left(t, t^{-1}\right)$, and let $X_{n} \subseteq \mathbb{P}^{n} \times \mathbb{P}^{n}$ denote the closure of its image. Check that $X_{n}$ is a toric variety with $P_{X_{n}}=\Delta^{n}+\left(-\Delta^{n}\right)$, where $\Delta^{n} \subseteq \mathbb{Z}^{n+1}$ denotes the standard simplex, and $-\Delta^{n}$ its reflection through the origin. Show that $X_{2}$ is isomorphic to a blowup of $\mathbb{P}^{2}$ at three points, so $X_{2}$ is smooth, but that $X_{n}$ is not smooth for $n \geq 3$.

\section{TRopical aspects}

Our third ingredient is a bit of tropical geometry. The reader should see [Mik06] or [RGST05] for an overview, and [Kat09] for an advanced introduction to the subject. The first "tropical aspect" here involves term orders for subvarieties of the torus.

4.1. Initial ideals. Let $I$ be an ideal either in the polynomial $\operatorname{ring} S:=\mathbb{C}\left[x_{1}, \ldots, x_{n}\right]$. Suppose $u$ is a linear functional (regarded as an element of $\mathbb{R}^{n}$ ). Then $u$ induces an order on monomials. For $a \in \mathbb{Z}^{n}$, write $x^{a}:=x_{1}^{a_{1}} \cdots x_{n}^{a_{n}}$, and put $x^{a} \prec_{u} x^{b}$ if and only if $u(a)<u(b)$. Then the initial ideal $\operatorname{In}_{u}(I)$ is obtained from $I$ by taking the $\prec_{u}$-maximal summand of each element of $I$ (allowing ties.) If $I$ is a homogeneous ideal, then we may take $u \in N_{\mathbb{R}}:=\mathbb{R}^{n} / \mathbb{R}_{[n]}$. Impose an equivalence relation on $N$ by putting $u \sim v$ if and only if $\operatorname{In}_{u}(I)=\operatorname{In}_{v}(I)$. This is in fact a polyhedral fan, called the Gröbner fan of $I$ : see, for example, [Stu96] for details.

The special case that interests us here is when $I=I(V)$, the defining ideal of a linear variety $V$. Then $I$ is generated in degree 1 by $V^{\perp}$, and the linear elements: i.e., $I$ is the defining ideal of a linear subvariety $V \subseteq \mathbb{C}^{n}$ (or, rather, its restriction to $T^{n}$.) In that case, $I=\left(V^{\perp}\right.$ ), 
and Sturmfels notes in [Stu02] that the Gröbner fan is simply the (outer) normal fan to the matroid polytope.

To see why this should be the case, the key observation is that we can compute initial ideals using limits of one-parameter subgroups. Based on the explicit calculation in Example 3.4, suppose that $I=\left(V^{\perp}\right)$, and $u \in N$ is some lattice direction, which again we regard as a one-parameter subgroup of the torus by letting $u(t)=\left(t^{u_{1}}, \ldots, t^{u_{n}}\right)$. Then, for any vector $v=\sum_{i=1}^{n} c_{i} x_{i} \in V^{\perp}$, we have

$$
u\left(t^{-1}\right) \cdot v=\sum_{i=1}^{n} c_{i} t^{-u_{i}} x_{i}
$$

Since $u$ is only defined up to a multiple of the vector $e_{[n]}$, we may choose our representative so that $-u_{i} \geq 0$ for all $i$, and the largest coordinate(s) are equal to zero. Then computing the limit as $t \rightarrow 0$ leaves us with only the $\prec_{u}$-initial terms of $v$, so $\lim _{t \rightarrow 0} u\left(t^{-1}\right) \cdot V^{\perp}=\left(V_{u}\right)^{\perp}$, denoting the linear space defined by $\operatorname{In}_{u}(V)$ by $V_{u}$. Since $(t \cdot V)^{\perp}=t^{-1} \cdot V^{\perp}$ (see Exercise 5.2), we see also that $\lim _{t \rightarrow 0} u(t) \cdot V=V_{u}$.

Using Theorem 3.3, then, $u \sim v$ in the inner normal fan of $P_{\mathrm{M}}$ if and only if

$$
\lim _{t \rightarrow 0} u(t) \cdot V=\lim _{t \rightarrow 0} v(t) \cdot V \Longleftrightarrow V_{u}=V_{v},
$$

if and only if $u \sim v$ in the Gröbner fan of $I$.

Since the cones of a normal fan are in bijection with polytope faces, the set of all the initial ideals is indexed by the faces of the matroid polytope.

Proposition 4.1. If $\mathrm{M}=\mathrm{M}(V)$ and $P_{\mathrm{M}_{u}}$ is any face of the polytope $P_{\mathrm{M}}$, then the degeneration matroid $\mathrm{M}_{u}$ equals $\mathrm{M}\left(V_{u}\right)$, where $V_{u}$ is the linear space defined by $\operatorname{In}_{u}(I(V))$.

4.2. Bergman fans. Now let us repeat the construction above where, this time, $I$ is an ideal in the coordinate ring of the torus, $\mathbb{C}\left[T^{n}\right]=\mathbb{C}\left[x_{1}^{ \pm 1}, \ldots, x_{n}^{ \pm 1}\right]$. Assume $I$ is a proper ideal generated in degree 1 . Then, from $\S 2.7$, the zero locus of such an ideal is a hyperplane complement $U(\mathcal{A})$ in a linear space $V$, where the matroid $\mathrm{M}(V)$ contains no loops. (Conversely, if $i$ is a loop in a linear matroid $\mathrm{M}(V)$, then $I(V)$ contains a unit, the variable $x_{i}$, which is to say $V \cap T^{n}=\emptyset$.) So by Proposition 4.1, the faces of the matroid polytope corresponding loopfree degenerations are in bijection with those initial ideals $\operatorname{In}_{u}(I(V))$ that define (nonempty) hyperplane complements. This motivates the following definition.

Definition 4.2. For a subvariety $X \subseteq T^{n}$ defined by an ideal $I$, its Bergman fan $\widetilde{B}(X)$ is the set of cones $\sigma_{u}$ in the Gröbner fan for which $\operatorname{In}_{u}(I)$ does not contain a monomial.

In the special case where $X=V \cap T^{n}$, we saw above that the Bergman fan depends only on the matroid $\mathrm{M}=\mathrm{M}(V)$, and accordingly we will denote it by $\widetilde{B}(\mathrm{M})$. This is the set of cones $\sigma_{u}$ of $\Sigma_{\mathbf{M}}$ for which the degeneration matroid $\mathbf{M}_{u}$ does not contain a loop. Theorem 2.1 gives another characterization, as well. Recall $\mathrm{M}_{u}$ contains a loop if and only if the face $u$ lies in the boundary of the standard simplex, so $\widetilde{B}(\mathrm{M})$ consists of (outer) normal cones to the faces of $\partial\left(d \cdot \Delta^{n-1}\right)-\partial P_{\mathrm{M}}$. In order to visualize it conveniently, we recall that the (outer) normal fan of a polytope consists of cones over the faces of the polar polytope $P_{\mathrm{M}}^{*}$. That is, $\widetilde{B}(\mathrm{M})$ is a cone over a polyhedral subcomplex of $P_{\mathrm{M}}^{*}$, which we denote $B_{\mathrm{M}}$. This is the Bergman complex of $\mathrm{M}$.

Example 4.3. Continuing Example 3.4, the defining ideal of $V$ is

$$
I=I(V)=\left(V^{\perp}\right)=\left(-x_{1}+x_{2}+x_{3},-x_{1}-x_{2}+x_{4}\right) .
$$




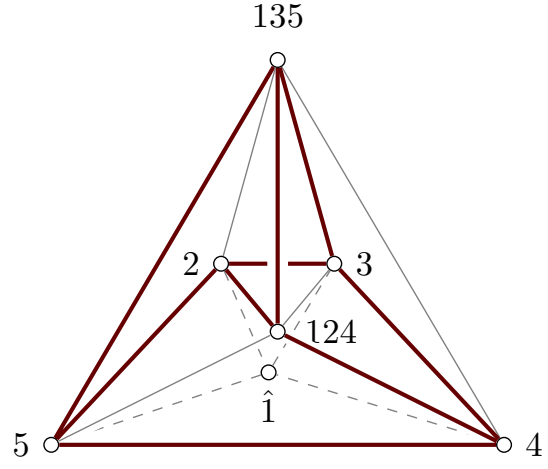

(a) $B_{\mathrm{M}}$ inside the Schlegel diagram of $P_{\mathrm{M}}^{*}$

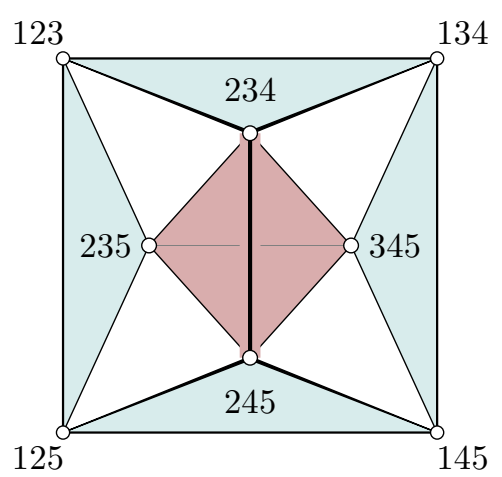

(b) The loopy subcomplex of $P_{\mathrm{M}}$

Figure 2. A Bergman complex and its dual, Example 2.8

Taking $u=(1,0,0,0)$, for example, and rewriting the subspaces in (3.1) as kernels, we find $\operatorname{In}_{u}(I)=\left(x_{1}, 2 x_{2}+x_{3}-x_{4}\right)$. By inspection the only vectors $u$ for which $\operatorname{In}_{u}(I)$ does not contain a variable are the nonnegative multiples of $(0,1,1,1),(1,0,1,1),(1,1,0,1)$, and $(1,1,1,0)$, and the Bergman fan consists of the bold rays in Figure 1(b).

Example 4.4. For the matroid of Examples 2.8 and 3.5, the subcomplex of $\partial P_{\mathrm{M}}$ consisting of faces in $\partial\left(3 \cdot \Delta^{4}\right)$ is shown in Figure 2(b). On the other hand, the polar polytope $P_{\mathrm{M}}^{*}$ has seven vertices, which lie in the directions $\left\{-e_{1}, e_{2}, e_{3}, e_{4}, e_{5}, e_{124}, e_{135}\right\}$. The Bergman complex $B_{\mathrm{M}}$ consists of the edges shown in bold in Figure 2(a).

Remark 4.5. Ardila and Klivans [AK06] show that the Bergman complex $B_{\mathrm{M}}$ is homeomorphic to the order complex of the open interval $(\hat{0},[n])$ in $L(\mathrm{M})$. The latter is known to be homeomorphic to a wedge of $\mu$ many $(d-2)$-spheres, where the number $\mu$ is, up to sign, the value of the Möbius function over the lattice: $\mu=(-1)^{d} \mu_{L(\mathrm{M})}(\hat{0},[n])$. Hacking finds an interesting generalization of this in [Hac08]. The number $\mu$ is also the top Betti number of $\mathbb{P} U(\mathcal{A})$, if $\mathrm{M}$ is the matroid of a complex arrangement $\mathcal{A}$.

By Alexander duality, the subcomplex of $P_{\mathrm{M}}$ consisting of faces indexed by matroids with loops also has the homology of a wedge of $\mu$ spheres of dimension $n-d-1$. In the examples shown in Figures 1, 2(b), it can be seen that this "loopy subcomplex" is homotopic to a wedge of spheres. It would be interesting to know a direct proof.

4.3. Amoebas. For a subset $X \subseteq T^{n}$, its (classical) amoeba is defined to be the set

$$
A_{t}(X):=\left\{\left(\log _{t}\left|x_{1}\right|, \log _{t}\left|x_{2}\right|, \ldots, \log _{t}\left|x_{n}\right|\right): x \in X\right\} \subseteq \mathbb{R}^{n},
$$

where $t>1$ is the base of the logarithm. If $X$ is closed under the diagonal action of $\mathbb{C}^{*}$, then $A_{t}(X)$ is invariant under translation by the vector $e_{[n]}$, so $A_{t}(X)$ can be taken to be a subset of $\mathbb{R}^{n} / \mathbb{R} e_{[n]}$.

The logarithmic limit set, denoted $\log (X)$, is the limit (in the Hausdorff metric) of the amoebas $A_{t}(X)$ as $t \rightarrow \infty$. If $X$ is an algebraic subvariety of the torus, then a foundational result in tropical geometry from [Stu02, §9] says that points in $\log (X)$ are indexed by initial ideals defining nonempty varieties. 


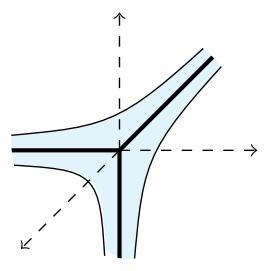

Figure 3. Amoeba and logarithmic limit set for $V$ with $\mathrm{M}(V)=U_{2,3}$

Theorem 4.6 ([Stu02]). For a subvariety $X$ of $T_{0}^{n-1}$, the set $-\log (X)$ equals the support of the Bergman fan, $|\widetilde{B}(X)|$. In particular, for a hyperplane complement $\mathbb{P} U \subseteq T_{0}^{n-1}$, we have $-\log (\mathbb{P} U)=\left|\widetilde{B}_{\mathrm{M}(V)}\right|$.

The tropicalization of $X$ consists of the set $|\widetilde{B}(X)|$, together with some additional (integer) data which we will ignore here. In the case where $X$ is linear, it is clear what information about $X$ is preserved in passing to its tropicalization: while an amoeba $A_{t}(X)$ depends on the equations defining $X$, the logarithmic limit set depends only on the underlying matroid.

Example 4.7. Consider a plane $V$ with $\mathrm{M}(V)=U_{2,3}$. Then

$$
A_{t}(\mathbb{P} U)=\left\{\left(\log _{t}|z|, \log _{t}|w|, \log _{t}|z-w|\right):[z: w] \in \mathbb{P} U(\mathcal{A})\right\} \subseteq \mathbb{R}^{3} / \mathbb{R}(1,1,1)
$$

An amoeba and logarithmic limit set for $\mathbb{P} U$ are shown in Figure 3.

\section{Compactifications}

If $\mathcal{A}$ is an arrangement, we will say $Y$ is a compactification of $\mathcal{A}$ if $Y$ is a complete complex variety and $\mathbb{P} U(\mathcal{A})$ is a dense open subset of $Y$. Recall that a Zariski open subset is dense if and only if it is dense in the complex topology, and complete varieties are compact in the complex topology. Of course, one way to produce a compactification is to take the closure of the embedding of $\mathbb{P} U(\mathcal{A})$ in some complete variety, and we will do this here. Since arrangement complements sit naturally as subvarieties of tori, it makes sense to consider their closures in various toric varieties. We will give some examples before considering discussing the general theory in the next section.

5.1. The reciprocal plane. Of course, $\mathbb{P} V$ is a compactification of $\mathcal{A}$, obtained from embedding the complement in $\mathbb{P}^{n-1}$. A more interesting example with the same ambient space can be obtained by letting $i: T_{0}^{n} \rightarrow T_{0}^{n}$ denote the inverse map in the torus, and letting

$$
Y(\mathcal{A})=\overline{i(\mathbb{P} U(\mathcal{A}))} \subseteq \mathbb{P}^{n-1} .
$$

(Regarded as a rational map, $i: \mathbb{P}^{n-1} \rightarrow \mathbb{P}^{n-1}$ is called the standard Cremona transformation.) This construction has been studied in a number of papers: see, for example, [PS06, ST09, Loo03, HT03, SSV11]. Recently it appeared in the work of Huh and Katz on the log-concavity of the coefficients of the characteristic polynomial (in arbitrary characteristic): see [HK11] and [Len11].

In particular, the homogeneous coordinate ring of $Y(\mathcal{A})$ equals $\mathbb{C}\left[1 / f_{i}: 1 \leq i \leq n\right]$. To describe this as a quotient of a polynomial ring, continue to let $S:=\mathbb{C}\left[x_{1}, \ldots, x_{n}\right]$ denote the homogeneous coordinate ring of $\mathbb{P}^{n-1}$. The inclusion $Y(\mathcal{A}) \subseteq \mathbb{P}^{n-1}$ induces a surjective homomorphism

$$
S \rightarrow \mathbb{C}\left[1 / f_{i}: 1 \leq i \leq n\right]
$$


sending $x_{i}$ to $1 / f_{i}$, for $1 \leq i \leq n$. Let $I(\mathcal{A})$ denote the kernel of the map (5.2).

To describe generators of $I(\mathcal{A})$, let $\operatorname{supp}(c)=\left\{i \in[n]: c_{i} \neq 0\right\}$ for $c \in \mathbb{C}^{n}$, and let

$$
r_{c}:=\sum_{i \in \operatorname{supp}(c)} c_{i} \prod_{j \in \operatorname{supp}(c)-\{i\}} x_{j} .
$$

If $c \in V^{\perp}$, then $\sum_{i=1}^{n} c_{i} f_{i}=0$, so $r_{c} \in I(\mathcal{A})$. A set of generators for $I(\mathcal{A})$ is given by selecting those $r_{c}$ for which $c \in V^{\perp}$ and $\operatorname{supp}(c)$ is minimal (the circuits of $\mathcal{A}$ ). It is known that $S / I(\mathcal{A})$ is Cohen-Macaulay, and that it has a Gröbner basis indexed by the broken circuit complex [PS06]. Questions about syzygies of $I(\mathcal{A})$, such as degrees of minimal sets of generators and Castelnuovo-Mumford regularity, seem to be difficult in general: see [ST09]. Sanyal, Sturmfels and Vinzant give a matroidal condition that characterizes those subsets of the equations (5.3) determine $Y(\mathcal{A})$ set-theoretically in [SSV11, Prop. 21].

Example 5.1. If $d=\operatorname{dim}(V)=2$ and any two coordinates of $f: V \rightarrow \mathbb{C}^{n}$ are linearly independent, then $\mathrm{M}(V) \cong U_{2, n}$ (Example 2.7). The circuits of the matroid consist of all three-element subsets of $[n]$, and the ideal $I(\mathcal{A})$ is generated by quadrics. By general theory (or just hands-on linear algebra), $\left(\begin{array}{c}n-1 \\ 2\end{array}\right)$ quadrics are required. Since $\mathbb{P} V$ has codimension $n-2$ in $\mathbb{P}^{n-1}$, so does $Y(\mathcal{A})$. Hence the depth (and projective dimension) of $I(\mathcal{A})$ are equal to $n-2$.

More generally, if $\mathrm{M}(V) \cong U_{d, n}$, a uniform matroid with arbitrary parameters, then again the syzygies of $I(\mathcal{A})$ can be understood completely. $I(\mathcal{A})$ is generated by $\left(\begin{array}{c}n-1 \\ k\end{array}\right)$ generators of degree $d$, and $I(\mathcal{A})$ has a linear resolution which is an Eagon-Northcott complex: see [DGT12, $\S 4.4]$.

5.2. Visible contours. The reciprocal plane lacks some features that one normally expects from a compactification: in particular, $Y(\mathcal{A})$ is not smooth, in general. (The singular locus is described in [SSV11].) The following construction is an improvement.

Recall that, provided the matroid $\mathrm{M}(V)$ is connected, the torus orbit of $V$ in $\mathrm{Gr}_{d, n}$ given by $a_{V}(t)=t \cdot V$ was isomorphic to $T_{0}^{n-1}$, and we saw in the previous section that the orbit closure was a toric variety $X_{\mathrm{M}}$ given, abstractly, by the matroid $\mathrm{M}=\mathrm{M}(V)$ (Theorem 3.3).

Since the arrangement complement $\mathbb{P} U(\mathcal{A})$ is given as a subspace of $T_{0}^{n-1}$, it makes sense to consider its closure inside $\mathrm{Gr}_{d, n}$. Instead of doing this directly, though, we want to use the reciprocal embedding instead, and define

$$
Y_{\mathrm{vc}}(\mathcal{A})=\overline{a_{V} \circ i(\mathbb{P} U(\mathcal{A}))} \subseteq \mathrm{Gr}_{d, n} .
$$

This is the visible contours compactification from [Kap93]. It is also called the tropical compactification in [FS05]; however, we will avoid the term here, since there are various tropical compactifications in the sense of Tevelev [Tev07]. We leave it as an exercise to the reader to find the appropriate modifications in the case where $\mathrm{M}(V)$ has more than one connected component.

To see why the inverse map should appear here, consider replacing $V$ by another subspace in the same orbit, $V^{\prime}:=t \cdot V$, for some $t \in T_{0}^{n-1}$. The hyperplane complements are related by $\mathbb{P} U^{\prime}:=t \cdot \mathbb{P} U$. Then

$$
\begin{aligned}
a_{V^{\prime}} \circ i\left(\mathbb{P} U^{\prime}\right) & =a_{t V} \circ i(t \cdot \mathbb{P} U) \\
& =a_{V} \circ t i(t \cdot \mathbb{P} U) \\
& =a_{V} \circ i(\mathbb{P} U),
\end{aligned}
$$

so the construction of $Y_{\mathrm{vc}}(\mathcal{A})$, as a subvariety of the Grassmanian, is independent of our choice of subspace from the orbit $T_{0}^{n-1} \cdot V$.

We leave some straightforward assertions about the inverse map as an exercise: 


\section{Exercise 5.2.}

- For $t \in T_{0}^{n-1}$ and $V \in \mathrm{Gr}_{d, n}$, we have $(i(t) \cdot V)^{\perp}=t \cdot V^{\perp}$.

- If $X$ is a toric variety with torus $T$, define a toric variety $X^{-1}$ whose underlying variety is also $X$, but the action of $T$ on $X^{-1}$ is constructed from the action on $X$ by letting $t \cdot x=i(t) x$. Show that $\Sigma_{X^{-1}}=-\Sigma_{X}$. If $X$ is projective, show that $P_{X^{-1}}=-P_{X}$. Decide when $X$ and $X^{-1}$ are isomorphic as toric varieties.

- For any matroid $\mathrm{M}$, check the weight polytope of $X_{\mathrm{M}}^{-1}$ is $P_{\mathrm{M}^{*}}$ (see Exercise 2.6.)

Of course we could also define $Y_{\mathrm{vc}}(\mathcal{A})$ as the closure of the hyperplane complement in the toric variety $X_{\mathrm{M}}^{-1}$, since

$$
\mathbb{P} U(\mathcal{A}) \hookrightarrow X_{\mathrm{M}}^{-1} \subseteq \mathrm{Gr}_{d, n}
$$

The hyperplane complement has codimension $n-d$ in $X_{\mathrm{M}}^{-1}$, and its closure only intersects some of the torus orbits. Those orbits have a nice description.

Theorem 5.3 ([FS05]). For all $\sigma \in \Sigma_{\mathrm{M}}$, we have $O(\sigma) \cap Y_{\mathrm{vc}}(\mathcal{A}) \neq \emptyset$ if and only if $\sigma \in \widetilde{B}(\mathrm{M})$. That is, the smallest toric variety in $X_{\mathrm{M}}^{-1}$ that contains $Y_{\mathrm{vc}}(\mathcal{A})$ is $X_{\widetilde{B}(\mathrm{M})}$.

We note that $\widetilde{B}(\mathrm{M})$ is not a complete fan, so our minimal compactifying toric variety $X_{\widetilde{B}(\mathrm{M})}$ is not, itself, compact. In [Tev07, Thm. 1.5], Tevelev shows that linear spaces are schön, which means in particular that the restriction of the multiplication map $T_{0}^{n-1} \times Y_{\mathrm{vc}}(\mathcal{A}) \rightarrow X_{\widetilde{B}(\mathrm{M})}$ is smooth. It follows that $Y_{\mathrm{vc}}(\mathcal{A})$ is smooth if and only if $X_{\widetilde{B}(\mathrm{M})}$ is smooth, and in general, $Y_{\mathrm{vc}}(\mathcal{A})$ has at most toroidal singularities. Examples show that the Bergman fan need not be simplicial, so the visible contours compactification is not, in general, smooth: for a family of examples, see [DD12]. Here is one from the literature.

Example 5.4. Let $\mathcal{A}$ be a hyperplane arrangement consisting of the six face planes of a cube in $\mathbb{P}^{3}$ : for example, let $V$ be the image in $\mathbb{C}^{4}$ of

$$
f(z, u, v, w)=(z+u, z-u, z+v, z-v, z+w, z-w) .
$$

Then $\mathcal{B}_{\mathrm{M}(\mathcal{A})}$ is a nonsimplicial 2-complex, described explicitly in [FS05, Ex. 2.8].

Exercise 5.5. Note that $\mathrm{Gr}_{1, n}=\mathbb{P}^{n-1}$, and find a family of compactifications that interpolates between the reciprocal plane (5.1) and the visible contours compactification (5.4).

Example 5.6. For $\mathrm{M}=U_{2,4}$, we saw that $\widetilde{B}(\mathrm{M})$ consists of the four rays shown in bold in Figure 1(b), and Theorem 5.3 can be verified directly. For any $t \in T_{0}^{3}$, the plane $i(t) \cdot V$ is the row space of

$$
\left(\begin{array}{cccc}
t_{1}^{-1} & 0 & t_{3}^{-1} & t_{4}^{-1} \\
0 & t_{2}^{-1} & -t_{3}^{-1} & t_{4}^{-1}
\end{array}\right) .
$$

On the other hand, a point $[z: w] \in \mathbb{P}^{1}$ is in $\mathbb{P} U$ provided that $z, w \neq 0$ and $z \neq \pm w$. Its image under $a_{V} \circ i$, using (5.5), is the set of solutions to

$$
\begin{aligned}
& -z x_{1}+w x_{2}+(z-w) x_{3}=0 \\
& -z x_{1}-w x_{2}+(z+w) x_{4}=0 .
\end{aligned}
$$

In this case, we simply have $Y_{\mathrm{vc}}(\mathcal{A}) \cong \mathbb{P}^{1}$, since the boundary points are obtained by letting $z=0, w=0, z=w$, or $z=-w$. In each case, the linear space of solutions is not contained in a coordinate hyperplane, so its matroid has no loop, and it lies in a torus orbit indexed by a shaded face of $P_{\mathrm{M}}$ in Figure 1. 
5.3. Maximum likelihood, master functions and critical points. Before moving to the next construction, we digress slightly in order to mention another, closely related variety. For a subspace $V$, define

$$
\Gamma_{V}=\left\{(x, y) \in T_{0}^{n-1} \times \mathbb{C}^{n}: y \in x \cdot V^{\perp}\right\},
$$

noting again that $x \cdot V^{\perp}=(i(x) \cdot V)^{\perp}$. Pulling back the projection onto the first factor along the inclusion of the hyperplane complement gives a new variety $\mathcal{S}(\mathcal{A})$, the variety of critical points:

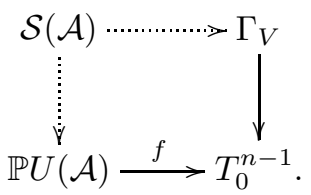

To justify the name, we should restrict our attention to lattice points $m \in \mathbb{Z}^{n}$. Then $(x, m) \in$ $\mathcal{S}(\mathcal{A})$ if and only if $x$ is a critical point of the master function $\prod_{i=1}^{n} f_{i}^{m_{i}}$ (see Remark 2.14). The second factor of $(5.6)$ is stable under the diagonal action of $\mathbb{C}^{\times}$: let $\mathfrak{X}(\mathcal{A})$ denote the closure of the quotient of $\mathcal{S}(\mathcal{A})$ in $\mathbb{P}^{n-1} \times \mathbb{P}^{n-1}$ : this is compact, but not smooth. It is known to be Cohen-Macaulay for all arrangements $\mathcal{A}$, but not, in general, arithmetically Cohen-Macaulay ([CDFV11]).

If we let $\mathbb{P} U^{\perp}=\mathbb{P} V^{\perp} \cap T_{0}^{n-1}$, the complement of the dual arrangement, then $\mathcal{S}(\mathcal{A}) / \mathbb{C}^{\times}$ contains a simple-looking dense, open subset:

$$
\begin{aligned}
\left(\mathcal{S}(\mathcal{A}) / \mathbb{C}^{\times}\right) \cap T & =\left\{(x, x y) \in T: x \in \mathbb{P} U, y \in \mathbb{P} U^{\perp}\right\}, \\
& \cong \mathbb{P} U \times \mathbb{P} U^{\perp},
\end{aligned}
$$

where $T:=T_{0}^{n-1} \times T_{0}^{n-1}$. With this in mind, we see that the compactification $\mathfrak{X}(\mathcal{A})$ is the closure of $\mathbb{P} U \times \mathbb{P} U^{\perp}$ in a toric variety isomorphic to $\mathbb{P}^{n-1} \times \mathbb{P}^{n-1}$ with a nonstandard torus action. The automorphism $T \rightarrow T$ given by $(x, y) \mapsto(x, x y)$ induces a linear automorphism of the character lattice $M=\mathbb{Z}^{n-1} \times \mathbb{Z}^{n-1}$, and the weight polytope of the toric variety is just the image of $\Delta^{n-1} \times \Delta^{n-1}$ under this map. We leave the details to the reader.

The critical points of a master function are interesting from various points of view, including algebraic statistics, mathematical physics, and the theory of hyperplane arrangements: see, in particular, [CHKS06, DGS12]. Some remarkable results about critical point varieties about more general very affine varieties appears in [Huh12].

By regarding the critical point variety as a compactification of a product of projective linear spaces, we see some symmetry between the underlying matroid and its dual that is not immediately apparent from the perspective of critical points of master functions. In particular, $\mathfrak{X}(\mathcal{A}(V))$ and $\mathfrak{X}\left(\mathcal{A}\left(V^{\perp}\right)\right)$ are both compactifications of $\mathbb{P} U \times \mathbb{P} U^{\perp}$, while their respective ambient toric varieties differ by the involution exchanging the factors.

5.4. Wonderful models. In 1995, De Concini and Procesi [DCP95] studied a family of compactifications obtained by iteratively blowing up the projective space $\mathbb{P} V$ along (proper transforms of) linear subspaces, in increasing order of dimension. Their compactification can be obtained in several different ways, and it has some very desirable properties. In particular, the boundary of $\mathbb{P} U(\mathcal{A})$ is well-behaved: it is a union of hypersurface components, each one isomorphic to $\mathbb{P}^{d-1}$. These components intersect with normal crossings, which is to say that the neighbourhood of a boundary point looks locally like an intersection of coordinate hyperplanes. 
The number of boundary components and the way in which they intersect depends on some interesting combinatorics, which we will now describe. A good expository resource is provided by [Fei05]. A detailed analysis and abstraction of the relevant combinatorics appears in [FK04].

Definition 5.7. Let $L$ be a partially ordered set with unique minimal element $\hat{0}$. A subset $G \subseteq L-\{\hat{0}\}$ is a building set if, for every $X \in L$, we have an order-isomorphism

$$
[\hat{0}, X] \cong \prod_{Y \in \max (G \cap[\hat{0}, X])}[\hat{0}, Y],
$$

where, for a set $S \subseteq L$, the notation "max $S$ " denotes the subset of maximal elements.

Lemma 5.8 (Prop. 2.5(1), [FK04]). Suppose $G$ is a building set, $Y \in L$, and $X \in G$. If $X \leq Y$, then $X \leq Y_{i}$ for a unique element $Y_{i} \in \max (G \cap[\hat{0}, Y])$.

The wonderful models are parameterized by building sets in the lattice of flats, $L(\mathrm{M})$.

Example 5.9. Tautologically, $G=L_{\geq 1}(\mathrm{M})$ is a building set for $L(\mathrm{M})$. On the other hand, it is not hard to see that if $G$ is a building set, then it must be the case that $G \supseteq L_{\text {irr }}(\mathrm{M})$. By the discussion in $\S 2.2$, if $X$ is not irreducible, then $L(\mathrm{M} \mid X)=[\hat{0}, X]$ decomposes as a product of lower intervals in $L(\mathrm{M})$, from which it follows that $L_{\mathrm{irr}}(\mathrm{M})$ is, itself, a building set. So we see that $G_{\min }:=L_{\text {irr }}(\mathrm{M})$ and $G_{\max }:=L_{\geq 1}(\mathrm{M})$ are the unique maximal and minimal building sets, respectively, and building sets themselves form a poset under inclusion. See, for example, [GS12], where the authors consider families of building sets.

Let $\mathcal{A}$ be a hyperplane arrangement in $V \subseteq \mathbb{C}^{n}$, and let $G \subseteq L(\mathrm{M}(V))$ be a building set. For each $X \in G$, the coordinate projection $\mathbb{C}^{n} \rightarrow \mathbb{C}^{X}$ from $\S 2.2$ induces a rational map $p_{X}: \mathbb{P}^{n-1} \rightarrow \mathbb{P}^{|X|-1}$ which is regular (i.e., defined) on the torus $T_{0}^{n-1}$. Let

$$
p: \mathbb{P}^{n-1} \rightarrow \prod_{X \in G} \mathbb{P}^{|X|-1}
$$

be the map whose $X$ th coordinate is $p_{X}$. Again, this is regular on $T_{0}^{n-1}$, so its restriction to $\mathbb{P} U(\mathcal{A})$ is as well. By definition, the image of $V$ under coordinate projection is $V_{X}$, so we may factor the restriction of $p$ as follows:

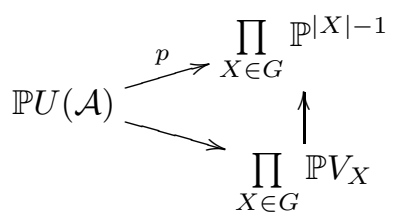

Since $\mathrm{M}$ is connected, the maximal flat $[n]$ is in $G$, so $\mathbb{P}^{n-1}$ and $\mathbb{P} V$ are factors in the top and bottom products, respectively. Then the maps in (5.9) are all injective, so the next definition makes sense.

Definition 5.10. The De Concini-Procesi wonderful compactification of $\mathcal{A}$ with building set $G$ is

$$
Y_{\text {wnd }}(\mathcal{A}, G)=\overline{p(\mathbb{P} U(\mathcal{A}))} \subseteq \prod_{X \in G} \mathbb{P} V_{X}
$$

The boundary components in $Y_{\text {wnd }}(\mathcal{A}, G)$ are indexed by the building set $G$. It remains to say which boundary components intersect, and we will see that this depends only on the matroid of $\mathcal{A}$ and the choice of building set. 
Note that a building set $G \subseteq L(\mathrm{M})$ necessarily contains all singleton flats $\{i\}$, since oneelement matroids are connected.

Definition 5.11. Let $G \subseteq L$ be a building set in a lattice $L$. A subset $S \subseteq G$ is a nested set for $G$ provided that, if $X_{1}, \ldots, X_{k}$ are pairwise incomparable elements of $S$, and $k \geq 2$, then the join $X_{1} \vee \cdots \vee X_{k} \notin G$. Let $\mathcal{N}(G)$ denote the set of all nested sets for $G$.

Example 5.12. Certainly if $S \subseteq G$ is a chain, it is nested. If $G=G_{\max }$, the maximal building set, then a nested set cannot have incomparable elements, so in this case $\mathcal{N}(G)$ is simply the set of chains in $L(\mathrm{M})$. On the other hand, if $G=G_{\min }$, then a nested set $S$ can contain incomparable elements, provided that their join is not irreducible.

Exercise 5.13. For the Boolean arrangement $\mathcal{C}_{n}$, the maximal building set consists of all nonempty subsets of $[n]$. Check that maximal nested sets for $G_{\max }$ are indexed by permutations of $[n]$. On the other hand, $G_{\min }$ consists of just the rank-1 flats (hyperplanes). Describe the nested sets in this case.

Exercise 5.14. If $\mathrm{M}$ is connected, the maximal flat $[n]$ is in every building set. Let $\mathcal{N}_{0}(G)$ denote the nested sets that do not contain $[n]$. Check that $\mathcal{N}(G)$ and $\mathcal{N}_{0}(G)$ are simplicial complexes on the building set $G$ and $G-\{[n]\}$, respectively. Show that $[n]$ is in every maximal nested set, so $\mathcal{N}(G)$ is a cone over $\mathcal{N}_{0}(G)$ at the vertex $[n]$.

These definitions were made to keep track of the incidence structure in the boundary of the compactification.

Theorem $5.15(\S 4.2,[\mathrm{DCP} 95])$. The boundary $Y_{\mathrm{wnd}}(\mathcal{A}, G)-p(\mathbb{P} U(\mathcal{A}))$ is a union of divisors $\left\{D_{X}: X \in G-\{[n]\}\right\}$. For a subset $S \subseteq G-\{[n]\}$, the intersection $\bigcap_{X \in S} D_{X}$ is nonempty if and only if $S$ is a nested set for $G$, in which case the intersection is transversal and irreducible.

It should be mentioned that De Concini and Procesi's construction is more general, allowing an arbitrary union of linear subspaces (over any infinite field) in place of an arrangement of hyperplanes; however, it is worth singling out this special case, since it arises most often, and the theory is somewhat simpler. Here we give the projective version of their construction: an affine variation gives a closure of $U(\mathcal{A})$ with an additional divisor indexed by the maximal flat.

Example 5.16. Consider the matroid of Example 2.8 again. In this case, we have $G_{\min }=$ $\{1,2, \ldots, 5,124,135,12345\}$. A realization of the complex $\mathcal{N}_{0}\left(G_{\min }\right)$ is given by the bold edges shown in Figure 4(b).

Here are some facts about nested sets which we will use in the next section. Although nested sets need not be chains, in general, they behave like chains in the following ways.

Lemma 5.17 (Prop. 2.8(2), [FK04]). Suppose $Y_{1}, \ldots, Y_{k}$ are pairwise incomparable elements in a nested set for a building set $G$, and $Y=Y_{1} \vee \cdots \vee Y_{k}$. Then the elements of $\max (G \cap[\hat{0}, Y])$ are precisely $\left\{Y_{1}, \ldots, Y_{k}\right\}$.

Lemma 5.18. Let $G \subseteq L(\mathrm{M})$ be a building set, and $S \in \mathcal{N}(G)$ a nested set for $G$. For each $i \in[n]$, let $S_{i}=\{X \in S: i \in X\}$. Then, for any $X \in L(\mathrm{M})$, the collection of nested sets $\left\{S_{i}: i \in X\right\}$ has a unique minimal element under inclusion; i.e., there exists some $i_{0} \in X$ for which $S_{i_{0}} \subseteq S_{i}$, for all $i \in X$.

Proof. We can assume $X \neq \hat{0}$, for which the result is trivial. Suppose the claim is false: that is, for each $i \in X$, there is a flat $Y_{i} \in S_{i}-\bigcap_{j \in X} S_{j}$. Consider the set $\left\{Y_{i}: i \in X\right\} \subseteq L(\mathrm{M})$, and let $\left\{Y_{i_{1}}, Y_{i_{2}}, \ldots, Y_{i_{k}}\right\}$ denote its subset of maximal elements (a pairwise incomparable set). We 
must have $k \geq 2$, since otherwise the flats $\left\{Y_{i}\right\}$ would form a chain, in which case the maximal element $Y_{i_{1}} \in S_{j}$ for all $j$, a contradiction.

By the nested set property, the join

$$
Y:=Y_{i_{1}} \vee \cdots \vee Y_{i_{k}}
$$

is not an element of $G$. Since $Y=\bigvee_{i \in X} Y_{i}$ as well, and $i \in Y_{i}$ for each $i \in X$, we see $X \leq Y$. Since $G$ is a building set, and $X \in G$, it must be the case that $X \leq Y_{i_{j}}$ for some $j$, by Lemma 5.17. But then $Y_{i_{j}} \in S_{i}$ for all $i$, a contradiction.

Lemma 5.19. The nonempty sets $S_{i}$ are chains. For every $X \in S$, there is some $i$ for which $X=\min S_{i}$.

Proof. Suppose $X$ and $Y$ are incomparable elements of $S$, and $X \in S_{i}$ for some $i$. The flat $\{i\}$ is irreducible, hence in $G$. Since $\{i\} \leq X$, we must have $\{i\} \not \subset Y$, by Lemma 5.8. That is, which is to say $Y \notin S_{i}$.

To check the second claim, suppose some $X \in S$ is not minimal in any chain $S_{i}$. That is, for all $i \in X$, there exists some $Y_{i} \in S_{i}$ for which $Y_{i}<X$. Then $X \leq \bigwedge_{i \in X} Y_{i}$, so $X \leq Y_{i}$ for some $i$ by Lemma 5.17, a contradiction.

Clearly if $X \in S_{i}$, then $Y \in S_{i}$ too, for all $Y \in S$ with $Y \geq X$. So we see, in particular, that, if we draw edges between comparable elements of a nested set $S$, the graph we obtain is a forest, with the leaves at the bottom.

For any nested set $S$, define an equivalence relation on the set $\bigvee_{X \in S} X \subseteq[n]$ by letting $i \sim_{S} j$ if and only if $S_{i}=S_{j} \neq \emptyset$. The corresponding partition has $|S|$ blocks.

Lemma 5.20. Let $S \in \mathcal{N}(G)$ be a nested set. Let $S=\left\{X_{1}, \ldots, X_{r}\right\}$ be any linear extension. The sets

$$
E_{k}:=\bigvee_{i=1}^{k} X_{i}-\bigvee_{i=1}^{k-1} X_{i} \subseteq[n]
$$

for $1 \leq k \leq r$ are all nonempty, and they are the blocks of the partition $\sim_{S}$.

Proof. First, we check that $E_{k}$ is nonempty for all $k$. If not, then $\bigvee_{i=1}^{k} X_{i}=\bigvee_{i=1}^{k-1} X_{i}$, which means $X_{k} \leq \bigvee_{i=1}^{k-1} X_{i}$. By Lemma 5.17, then $X_{k} \leq X_{i}$ for some $i \leq k-1$, which would contradict having chosen a linear extension.

Our linear extension gives a total order to each subset $S_{j}$ of $S$. With respect to this order, $j \in E_{k}$ if and only if $\min S_{j}=k$. Clearly if $i \sim_{S} j$, the sets $S_{i}=S_{j}$ have the same minimum, so each $E_{k}$ is a union of one or more blocks of $\sim_{S}$. Since there are only $r=|S|$ blocks, though, it follows each $E_{k}$ is equal to exactly one of them.

In the other direction,

Lemma 5.21 (Prop. 2.8(3), [FK04]). For any chain of flats $F_{1}<F_{2}<\cdots<F_{d}=[n]$ in an intersection lattice $L(\mathrm{M})$, for any building set, there exists a nested set $S \in \mathcal{N}(G)$ and a linear extension $S=\left\{X_{1}, \ldots, X_{d}\right\}$ for which $F_{k}=\bigvee_{i=1}^{k} X_{i}$, for all $1 \leq k \leq d$.

5.5. Wonderful models II. Once again, our compactification took place in a projective toric variety. Let $X_{\mathrm{wnd}}(\mathrm{M}, G)$ denote the closure of $T_{0}^{n-1}$, embedded diagonally in the product of projective spaces in (5.8). (Our notation reflects the fact that the toric variety depends only on the matroid of $\mathcal{A}$.)

For each flat $X \in L(\mathcal{A})$, let

$$
\Delta_{X}=\operatorname{conv}\left\{e_{i}: i \in X\right\}
$$


denote the weight polytope of $\mathbb{P}^{|X|-1}$. The weight polytope of $X_{\mathrm{wnd}}(\mathrm{M}, G)$ is the Minkowski sum $\sum_{X \in G} \Delta_{X}$, by Proposition 3.6 (see [FS05, §6]). It should also be mentioned that Ardila, Benedetti and Doker [ABD10] have found some remarkable results relating matroid polytopes and Minkowski sums of simplices, building on Postnikov's work on generalized permutohedra [Pos09]. In particular, they obtain a combinatorial formula for the degree of the toric variety $X_{\mathrm{M}}$.

In Section $\S 5.2$, we saw that we could replace our ambient, complete toric variety by a minimal one, indexed by the Bergman fan. Similarly, we can describe the minimal toric varieties that give the wonderful models, using a construction that first appeared in [FY04].

We continue to assume that $\mathrm{M}$ is connected. Let $G \subseteq L(\mathrm{M})$ be a building set. We construct a rational, polyhedral fan $\widetilde{\mathcal{N}}(\mathrm{M}, G)$ in $N_{\mathbb{R}}$ by taking the cone over the geometric realization of the complex $\mathcal{N}_{0}(G)$. That is, for each nested set $S \subseteq G$, define a cone

$$
\sigma_{S}=\mathbb{R}_{\geq 0} \operatorname{conv}\left\{e_{X}: X \in S\right\},
$$

and let $\tilde{\mathcal{N}}(\mathrm{M}, G)=\left\{\sigma_{S}: S \in \mathcal{N}(G)\right\}$. Since $\mathcal{N}(G)$ is a simplicial complex, $\widetilde{\mathcal{N}}(\mathrm{M}, G)$ is a simplicial fan. (From Exercise 5.14, we would have constructed the same fan by taking the cone over $\mathcal{N}(G)$ instead, since $e_{[n]}=0$ in the lattice $N$.)

Proposition 5.22. For any arrangement $\mathcal{A}$ and building set $G$, the toric variety $X_{\tilde{\mathcal{N}}(\mathrm{M}, G)}$ is a subvariety of $X_{\mathrm{wnd}}(\mathrm{M}, G)$.

Proof. An inclusion of normal toric varieties is given by an inclusion of toric fans, so let $\Sigma(G)$ denote the normal fan of $\sum_{X \in G} \Delta_{X}$. A straightforward argument with the weight polytope shows that $X_{\text {wnd }}(\mathrm{M}, G)$ is normal, so $\Sigma(G)$ is its toric fan. It is enough to check that each cone in the fan $\widetilde{\mathcal{N}}(\mathrm{M}, G)$ is contained in some cone of $\Sigma(G)$.

The normal fan of a Minkowski sum of polytopes is the coarsest common refinement of their respective normal fans: that is, two functionals $u, v \in N_{\mathbb{R}}$ lie in the same open cone of $\Sigma(G)$ if and only if they achieve their minimum on the same faces of $\Delta_{X}$, for each $X \in G$. Faces of a standard simplex are indexed by subsets of coordinates, so for a given $u \in N_{\mathbb{R}}$ and subset $I \subseteq[n]$, let

$$
\min _{I}(u)=\left\{i \in I: u_{i}=m, \text { where } m=\min \left\{u_{j}\right\}_{j \in I}\right\} .
$$

Then, translating the above, $u \sim v$ in $\Sigma(G)$ if and only if $\min _{X}(u)=\min _{X}(v)$ for all $X \in G$.

Now suppose that $u$ lies in the interior of a cone of $\widetilde{\mathcal{N}}(\mathrm{M}, G)$ indexed by a nested set $S \in \mathcal{N}(G)$, so $u=\sum_{X \in S} c_{X} e_{X}$ for some coefficients $c_{X}>0$. We need to show that the set $\min _{X}(u)$ is independent of the coefficients, for each $X \in G$. For this, let $S_{i}=\{X \in S: i \in X\}$, for each $i \in[n]$. Since $u_{i}=\sum_{X \in S_{i}} c_{X}$, we have $u_{i}=u_{j}$ if $S_{i}=S_{j}$, and $u_{i}<u_{j}$ if $S_{i} \subset S_{j}$, for all $i, j$.

By Lemma 5.18, the set $\left\{S_{i}: i \in X\right\}$ has a minimal element $S_{i_{0}}$, for some $i_{0} \in X$. But then $\min _{X}(u)=\left\{i \in X: S_{i}=S_{i_{0}}\right\}$, which depends only on the nested set $S$, so we are done.

Theorem 5.23. For any arrangement $\mathcal{A}$ and building set $G$,

$$
Y_{\text {wnd }}(\mathcal{A}, G)=\overline{\mathbb{P} U(\mathcal{A})} \subseteq X_{\tilde{\mathcal{N}}(\mathrm{M}, G)} .
$$

Moreover, the toric variety $X_{\widetilde{\mathcal{N}}(\mathrm{M}, G)}$ is minimal, in the sense that $\overline{\mathbb{P} U(\mathcal{A})}$ intersects every open torus orbit.

Once again, the compactifying ambient space depends only on the matroid. The situation is parallel to the one with $Y_{\mathrm{vc}}(\mathcal{A})$, since our small toric variety is not complete. However, 
TABLE 1. Arrangement compactifications in $\S 5$

\begin{tabular}{llccc}
\hline \multicolumn{2}{c}{ Compactification } & complete toric variety & weight polytope & reference \\
\hline $\mathbb{P} V$ & tautological & $\mathbb{P}^{n-1}$ & $\Delta^{n-1}$ & \\
$Y(\mathcal{A})$ & reciprocal plane & $\mathbb{P}^{n-1}$ & $-\Delta^{n-1}$ & $(5.1)$ \\
$Y_{\text {vc }}(\mathcal{A})$ & visible contours & $X_{\mathrm{M}}^{-1} \subseteq \mathrm{Gr}_{d, n}$ & $-P_{\mathrm{M}}\left(\cong P_{\mathrm{M}^{*}}\right)$ & $(5.4)$ \\
$Y_{\text {wnd }}(\mathcal{A}, G)$ & wonderful model & $X_{\text {wnd }}(\mathrm{M}, G) \subseteq \prod_{X \in G} \mathbb{P}^{|X|-1}$ & $\sum_{X \in G} \Delta_{X}$ & $(5.10)$ \\
\hline
\end{tabular}

\begin{tabular}{lclc}
\hline Compactification & minimal toric variety & toric fan & reference \\
\hline$Y_{\text {vc }}(\mathcal{A})$ & $X_{\widetilde{B}(\mathrm{M})} \subseteq X_{\mathrm{M}}^{-1}$ & Bergman fan & Theorem 5.3 \\
$Y_{\text {wnd }}(\mathcal{A}, G)$ & $X_{\widetilde{\mathcal{N}}(\mathrm{M}, G)} \subseteq X_{\text {wnd }}(\mathrm{M}, G)$ & nested set fan & Theorem 5.23 \\
\hline
\end{tabular}

Feichtner and Yuzvinsky [FY04] show that the cones of $\widetilde{\mathcal{N}}(\mathrm{M}, G)$ are unimodular, so the toric variety $X_{\widetilde{\mathcal{N}}(\mathrm{M}, G)}$ is always smooth, in contrast to $X_{\widetilde{B}(\mathrm{M})}$.

This construction also relates nicely to De Concini and Procesi's description of $Y_{\text {wnd }}(\mathcal{A}, G)$ as an iterated blowup, as shown in $[\mathrm{FY} 04, \S 6]$. Roughly speaking, one builds the fan $\widetilde{\mathcal{N}}(\mathrm{M}, G)$ by starting with the fan for $\mathbb{P}^{n-1}$ (Example 3.2), noting that $\tilde{\mathcal{N}}(\mathrm{M}, G$ ) always contains the rays through $e_{i}$, for $1 \leq i \leq n$. Then one adds rays $e_{S}$ and subdivides cones, for the remaining elements $S \in G$, in non-increasing order with respect to $L(\mathrm{M})$. Stellar subdivision in a fan corresponds to blowing up closed orbits in toric varieties: see, e.g., [CLS11, §3.3]. By blowing up $\mathbb{P}^{n-1}$ along proper transforms of coordinate subspaces, one obtains a complete toric variety; then $X_{\tilde{\mathcal{N}}(\mathrm{M}, G)}$ is the subvariety obtained by deleting the orbits not indexed by nested sets.

Our constructions so far are summarized in Table 5.5, where $V$ is a linear space, $\mathcal{A}=\mathcal{A}(V)$, and $\mathrm{M}=\mathrm{M}(V)$.

5.6. Comparisons. For a fixed arrangement, we now have a number of compactifications. In particular, if $G_{1} \subseteq G_{2}$ are two building sets for an intersection lattice $L(\mathrm{M})$, then the obvious projection map

$$
\prod_{X \in G_{2}} \mathbb{P} V_{X} \rightarrow \prod_{X \in G_{1}} \mathbb{P} V_{X}
$$

induces a map of wonderful compactifications $Y_{\text {wnd }}\left(\mathcal{A}, G_{2}\right) \rightarrow Y_{\text {wnd }}\left(\mathcal{A}, G_{1}\right)$. From De Concini and Procesi's original point of view, this map blows down the boundary divisor components indexed by $G_{2}-G_{1}$.

From the toric point of view, Feichtner and Müller prove in [FM05, Thm. 4.2] that the fan $\tilde{\mathcal{N}}\left(\mathrm{M}, G_{2}\right)$ is a refinement of the fan $\tilde{\mathcal{N}}\left(\mathrm{M}, G_{1}\right)$. More precisely, the former fan is obtained by stellar subdivisions of that latter. Geometrically, the map of toric varieties

$$
X_{\widetilde{\mathcal{N}}\left(\mathrm{M}, G_{2}\right)} \rightarrow X_{\widetilde{\mathcal{N}}\left(\mathrm{M}, G_{1}\right)}
$$

blows down the codimension- 1 torus orbits indexed by $G_{2}-G_{1}$. It follows that the support of the fan $\widetilde{\mathcal{N}}(\mathrm{M}, G)$ is independent of $G$ : that is,

$$
\left|\tilde{\mathcal{N}}\left(\mathrm{M}, G_{\text {min }}\right)\right|=|\tilde{\mathcal{N}}(\mathrm{M}, G)|=\left|\tilde{\mathcal{N}}\left(\mathrm{M}, G_{\text {max }}\right)\right|
$$


for any building set $G$.

A key discovery in [FS05] is the following (and we outline a proof below).

Theorem 5.24. For any matroid $\mathrm{M}$, the nested set fan $\tilde{\mathcal{N}}\left(\mathrm{M}, G_{\min }\right)$ refines the Bergman fan $\widetilde{B}(\mathrm{M})$.

This means that there is a natural map of toric varieties,

$$
X_{\widetilde{\mathcal{N}}\left(\mathrm{M}, G_{\min }\right)} \rightarrow X_{\widetilde{B}(\mathrm{M})},
$$

and a corresponding map of compactifications $Y_{\text {wnd }}(\mathcal{A}, G) \rightarrow Y_{\mathrm{vc}}(\mathcal{A})$ for any building set $G$ (by factoring through $G_{\min }=L_{\mathrm{irr}}(\mathrm{M})$ ).

It turns out that, if for every flat $X \in L_{\text {irr }}(\mathrm{M})$ it happens that $\mathrm{M} / X$ is also connected, then this subdivision can be accomplished without adding new vertices. If an even stronger condition holds, the two fans are actually equal:

Theorem 5.25 (Thm. 5.3, [FS05]). The fans $\widetilde{\mathcal{N}}\left(\mathrm{M}, G_{\min }\right)$ and $\widetilde{B}(\mathrm{M})$ are equal if and only if the matroid $(\mathrm{M} \mid Y) / X$ is connected for all pairs of flats $X \leq Y$, where $Y \in G_{\min }$.

Accordingly, if this matroid condition is satisfied, then the visible contours compactification $Y_{\mathrm{vc}}(\mathcal{A})$ is equal to the wonderful compactification given by the minimal building set. Before continuing with examples, we give another argument that makes use of nested set combinatorics.

Proof of Theorem 5.24. For any matroid M, we first show that each cone of $\widetilde{\mathcal{N}}\left(\mathrm{M}, G_{\text {min }}\right)$ is contained in a cone of $\widetilde{B}(\mathrm{M})$. It is enough to verify this for a cone $\sigma_{S}$ (defined in (5.13)) for a maximal nested set $S$, so suppose $u=\sum_{X \in S} c_{X} e_{X}$, where each coefficient $c_{X}>0$. Then $u_{i}=u_{j}$ if $i \sim_{S} j$, using the equivalence relation from $\S 5.4$. Let $\mathcal{F}(-u)=\left(F_{0}, \ldots, F_{k}\right)$ be the chain of subsets (2.1): then each set $F_{i}-F_{i-1}$ is a union of $\sim_{S}$-blocks. Let us temporarily assume that

$$
u_{i}=u_{j} \text { if and only if } i \sim_{S} j:
$$

i.e., each $F_{i}-F_{i-1}$ is a single block, and impose a total order on the nested set $S$ as follows. For flats $X, Y \in S$, recall $X=\min S_{i}$ and $Y=\min S_{j}$ for some $i, j$, by Lemma 5.19. In that case, put $X \prec Y$ if $u_{i}>u_{j}$. This is a linear extension of $S$, since if we had $X \leq Y$ in $L(\mathrm{M})$, then $Y \in S_{i}$ as well, whence $S_{j} \subseteq S_{i}$, and $u_{j} \geq u_{i}$. Write $S=\left\{X_{1}, \ldots, X_{d}\right\}$, numbering the flats in $\prec$-order. By our assumption that each set $F_{i}-F_{i-1}$ is a block of $\sim_{S}$, for $1 \leq i \leq k$, by Lemma 5.20 , we see $F_{i}=\bigvee_{j=1}^{i} X_{i}$, for each $i$.

This is a chain of flats of $\mathrm{M}$, so by Theorem 2.1, $-u$ achieves its minimum on a face $F=\mathrm{M}_{-u}$ indexed by a matroid without loops, so $u \in|\widetilde{B}(\mathrm{M})|$. Moreover, the chain $\mathcal{F}(-u)$ and face $F$ depended only on the set $S$ and not on the choice of $u$, provided ( $\star$ ) was satisfied. By convexity, the same is true for all $u$ in the interior of $\sigma_{S}$, so this cone lies in a cone of $\widetilde{B}(\mathrm{M})$.

It follows that $|\widetilde{\mathcal{N}}(\mathrm{M}, G)| \subseteq|\widetilde{B}(\mathrm{M})|$. To show that the supports are equal, consider a vector $u \in|\widetilde{B}(\mathrm{M})|$. By Theorem 2.1, elements of the chain $\mathcal{F}(-u)$ are flats of M. By Lemma 5.21, there exists a linear extension of a nested set $S=\left\{X_{1}, \ldots, X_{d}\right\}$ for the flats in the chain are $\left\{\bigvee_{i=1}^{k} X_{i}\right\}_{1 \leq k \leq d}$. By Lemma 5.20 and the first part of this proof, $u \in \sigma_{S}$.

Example 5.26. Continuing our usual Example 5.16, we see the nested set complex has one more vertex than the Bergman complex. Figure 4 compares the Bergman fan with the nested set complexes for the minimal and maximal building sets, $G_{\min }=L_{\mathrm{irr}}(\mathrm{M})$ and $G_{\max }=L_{\geq 1}(\mathrm{M})$. 


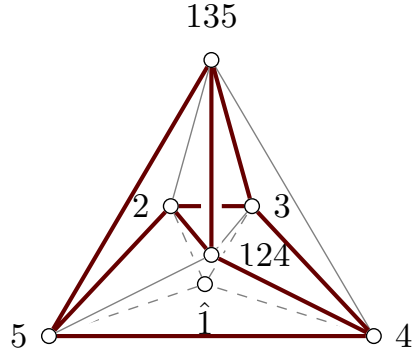

(a) $B_{\mathrm{M}}$

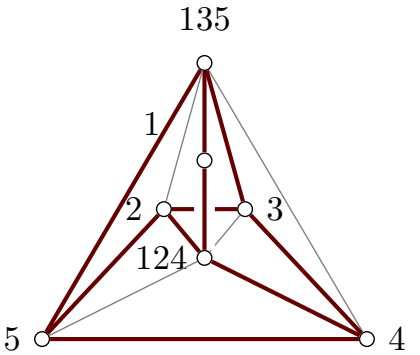

(b) $\Sigma\left(\mathcal{A}, G_{\min }\right)$

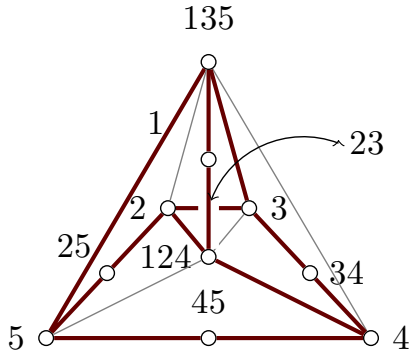

(c) $\Sigma\left(\mathcal{A}, G_{\max }\right)$

FiguRE 4. refinements of $B_{\mathrm{M}}$, Example 5.26

The toric variety for 4 (b) is obtained by blowing up $\mathbb{P}^{4}$ along two coordinate lines, then deleting the torus orbits of dimension $\leq 1$, as well as the 2-dimensional orbits indexed by non-nested pairs of rays. The closure of $\mathbb{P} U(\mathcal{A})$ inside is the blowup of $\mathbb{P}^{2}$ at the two triple intersections.

Then $Y_{\mathrm{vc}}(\mathcal{A})$ is obtained from $Y_{\mathrm{wnd}}\left(\mathcal{A}, G_{\mathrm{min}}\right)$ by blowing down the boundary component corresponding to the line through the two triple points, giving $\mathbb{P}^{1} \times \mathbb{P}^{1}$.

Example 5.27. The braid arrangements, Example 2.9, are particularly interesting. The original construction of $Y_{\text {wnd }}\left(\mathcal{A}, G_{\text {min }}\right)$ is the case $X=\mathbb{C}$ of a configuration space compactification of $F(X, d+1)$ due to Fulton and MacPherson [FM94]. Points in the arrangement complement for $V\left(A_{d}\right)$ are also in bijection with genus zero curves with $d+2$ marked points, and $Y_{\text {wnd }}\left(\mathcal{A}, G_{\min }\right)$ agrees with the Deligne-Knudsen-Mumford compactification.

The visible contours and wonderful compactifications agree (for $G=L_{\text {irr }}$ ), which we can check as follows. If $\pi_{X}$ is a partition of the set $[d+1]$, refinements $\pi_{Y}$ of $\pi_{X}$ are in bijection with partitions of the set of blocks of $\pi_{X}$. With this in mind, suppose we have flats $X<Y$ and $Y$ is irreducible. As a partition of the blocks of $\pi_{X}$, we see $\pi_{Y}$ still has only one block of size greater than one, so the interval $[X, Y]$ is order-isomorphic to the intersection lattice of another braid arrangement (of rank $r(Y)-r(X)$ ). Accordingly, $(\mathrm{M} \mid Y) / X$ is connected, and the claim follows by applying Theorem 5.25.

Example 5.28. If $\mathrm{M}(V)=U_{d, n}$, the uniform matroid, the situation is straightforward. Assume $d<n$; then $G:=L_{\text {irr }}\left(U_{d, n}\right)=\{\{i\}: 1 \leq i \leq n\} \cup\{[n]\}$. The nested sets are the subsets of size at most $d$, so the maximal cones in $\widetilde{\mathcal{N}}\left(U_{d, n}, G\right)$ are just the coordinate cones spanned by $d-1$-element subsets of $\left\{e_{i}: 1 \leq i \leq n\right\}$. This refines the Bergman fan, but no strictly coarser fan with the same support is possible, so $\widetilde{B}\left(U_{d, n}\right)=\widetilde{\mathcal{N}}\left(U_{d, n}, G\right)$, as we see for $U_{2,4}$ in Figure 1(b). Our ambient toric variety is $\mathbb{P}^{n-1}$, minus all coordinate subspaces of codimension $d$ and higher, and $Y_{\mathrm{vc}}(\mathcal{A})=Y_{\mathrm{wnd}}(\mathcal{A}, G)=\mathbb{P}^{d-1}$.

\section{Concluding Remarks}

By limiting our discussion to linear spaces in tori (that is, hyperplane complements), this tutorial stops short of "modern" tropical geometry. In particular, Tevelev's paper [Tev07] broadly generalizes the relationship we saw here between the Gel'fand-MacPherson construction, the Bergman fan, and the visible contours compactification. 
We have also neglected any discussion of intersection theory here, due to the constraints of time and space, although this is also an interesting part of the story above. We leave the last exercise as a possible starting point for further reading in this direction.

Exercise 6.1. We noted in Remark 4.5 that the support of the Bergman fan is a cone over a wedge of $\mu$ spheres of dimension $d-2$. From the discussion in $\S 5.6$, the same is true of the nested set fans $\widetilde{\mathcal{N}}(\mathrm{M}, G)$.

It is also known that the number $\mu$ is the degree of the reciprocal plane $Y(\mathcal{A})$, from [PS06, Lemma 2]: that is, $\mu$ is the number of points at which $Y(\mathcal{A})$ intersects a generic projective linear subspace $W$ in $\mathbb{P}^{n-1}$ of dimension $n-d$.

A basic idea of tropical intersection theory is that, under suitable conditions, one can compute an intersection number by intersecting tropicalizations: see, e.g., [Kat12] for details. Using Theorem 4.6, we know the tropicalizations of $Y(\mathcal{A})$ and $W$ are given by $\left|\widetilde{B}_{\mathrm{M}(V)}\right|$ and $-\left|\widetilde{B}_{\mathrm{M}(W)}\right|$, respectively. The linear space $W$ realizes the uniform matroid, so its Bergman fan was computed in Example 5.28.

Use the tropical intersection product (also known as the fan displacement rule) to give a "tropical" proof that $\mu$ is the degree of $Y(\mathcal{A})$.

Acknowledgement. The author is indebted to a number of people for helpful discussions about this material, in particular: Maria Angelica Cueto, Mike Falk, Eva Feichtner, June Huh, Eric Katz, Diane MacLagan, Kristin Shaw, and Jenia Tevelev.

\section{REFERENCES}

[ABD10] F. Ardila, C. Benedetti, and J. Doker, Matroid polytopes and their volumes, Discrete Comput. Geom. 43 (2010), no. 4, 841-854. MR2610473 (2012b:52026) 5.5

[AK06] F. Ardila and C. J. Klivans, The Bergman complex of a matroid and phylogenetic trees, J. Combin. Theory Ser. B 96 (2006), no. 1, 38-49. MR2185977 (2006i:05034) 1, 2.3, 2.1, 4.5

$\left[\mathrm{CDF}^{+}\right] \quad$ D. C. Cohen, G. Denham, M. J. Falk, H. K. Schenck, A. I. Suciu, H. Terao, and S. Yuzvinsky, Complex arrangements: algebra, geometry, topology, in preparation. 2.7

[CDFV11] D. C. Cohen, G. Denham, M. J. Falk, and A. Varchenko, Critical points and resonance of hyperplane arrangements, Canad. J. Math. 63 (2011), no. 5, 1038-1057. MR2866070 5.3

[CHKS06] F. Catanese, S. Hoşten, A. Khetan, and B. Sturmfels, The maximum likelihood degree, Amer. J. Math. 128 (2006), no. 3, 671-697. MR2230921 (2007m:13036) 5.3

[CLS11] D. A. Cox, J. B. Little, and H. K. Schenck, Toric varieties, Graduate Studies in Mathematics, vol. 124, American Mathematical Society, Providence, RI, 2011. MR2810322 (2012g:14094) 3, 5.5

[DCP95] C. De Concini and C. Procesi, Wonderful models of subspace arrangements, Selecta Math. (N.S.) 1 (1995), no. 3, 459-494. MR1366622 (97k:14013) 1, 5.4, 5.15

[DD12] E. Delucchi and M. Dlugosch, Bergman complexes of lattice path matroids, arXiv:1207.4700. 5.2

[DGS12] G. Denham, M. Garrousian, and M. Schulze, A geometric deletion-restriction formula, Adv. Math. 230 (2012), no. 4-6, 1979-1994. MR2927361 5.3

[DGT12] G. Denham, M. Garrousian, and Ş. Tohăneanu, Modular decomposition of the Orlik-Terao algebra of a hyperplane arrangement, Ann. Combin., to appear, arXiv:1211.4562. 5.1

[Fei05] E. M. Feichtner, De Concini-Procesi wonderful arrangement models: a discrete geometer's point of view, Combinatorial and computational geometry, MSRI Publ., vol. 52, Cambridge Univ. Press, Cambridge, 2005, pp. 333-360. MR2178326 (2006i:05178) 5.4

[FK04] E. M. Feichtner and D. N. Kozlov, Incidence combinatorics of resolutions, Selecta Math. (N.S.) 10 (2004), no. 1, 37-60. MR2061222 (2006k:06008) 5.4, 5.8, 5.17, 5.21

[FM05] E. M. Feichtner and I. Müller, On the topology of nested set complexes, Proc. Amer. Math. Soc. 133 (2005), no. 4, 999-1006 (electronic). MR2117200 (2006c:06005) 5.6

[FS05] E. M. Feichtner and B. Sturmfels, Matroid polytopes, nested sets and Bergman fans, Port. Math. (N.S.) 62 (2005), no. 4, 437-468. MR2191630 (2006j:05036) 1, 2.3, 2.1, 2.2, 5.2, 5.3, 5.4, 5.5, 5.6, 5.25

[FY04] E. M. Feichtner and S. Yuzvinsky, Chow rings of toric varieties defined by atomic lattices, Invent. Math. 155 (2004), no. 3, 515-536. MR2038195 (2004k:14009) 5.5, 5.5 
[FM94] W. Fulton and R. D. MacPherson, A compactification of configuration spaces, Ann. of Math. (2) 139 (1994), no. 1, 183-225. MR1259368 (95j:14002) 5.27

[GGMS87] I. M. Gel'fand, Mark Goresky, R. D. MacPherson, and V. V. Serganova, Combinatorial geometries, convex polyhedra, and Schubert cells, Adv. in Math. 63 (1987), no. 3, 301-316. MR877789 (88f:14045) 1, 2.3, 3.3

[GKZ94] I. M. Gel'fand, M. M. Kapranov, and A. V. Zelevinsky, Discriminants, resultants, and multidimensional determinants, Mathematics: Theory \& Applications, Birkhäuser Boston Inc., Boston, MA, 1994. MR1264417 (95e:14045) 3.6

[GM82] I. M. Gel'fand and R. D. MacPherson, Geometry in Grassmannians and a generalization of the dilogarithm, Adv. in Math. 44 (1982), no. 3, 279-312. MR658730 (84b:57014) 1

[GS] D. Grayson and M. Stillman, Macaulay2 - a software system for algebraic geometry and commutative algebra, available at http://www.math.uiuc.edu/Macaulay2. 3.5

[GS12] G. Gaiffi and M. Serventi, Families of building sets and regular wonderful models, arXiv:1210.7688. 5.9

[Hac08] P. Hacking, The homology of tropical varieties, Collect. Math. 59 (2008), no. 3, $263-273$. MR2452307 (2010c:14076) 4.5

[HT03] H. Horiuchi and H. Terao, The Poincaré series of the algebra of rational functions which are regular outside hyperplanes, J. Algebra 266 (2003), no. 1, 169-179. MR1994536 (2004k:13031) 5.1

[HK11] J. Huh and E. Katz, Log-concavity of characteristic polynomials and the Bergman fan of matroids, Math. Ann. 354 (2012), no. 3, 1103-1116. MR29830815.1

[Huh12] J. Huh, The maximum likelihood degree of a very affine variety, Compos. Math., to appear. arXiv: 1207.0553 .5 .3

[Kap93] M. M. Kapranov, Chow quotients of Grassmannians. I, I. M. Gel'fand Seminar, Adv. Soviet Math., vol. 16, Amer. Math. Soc., Providence, RI, 1993, pp. 29-110. MR1237834 (95g:14053) 5.2

[Kat09] E. Katz, A tropical toolkit, Expo. Math. 27 (2009), no. 1, 1-36. MR2503041 (2010f:14069) 4

[Kat12] E. Katz, Tropical intersection theory from toric varieties, Collect. Math. 63 (2012), no. 1, $29-44$. MR2887109 6.1

[Len11] M. Lenz, The f-vector of a realizable matroid complex is strictly log-concave, arXiv:1106.2944 5.1

[Loo03] E. Looijenga, Compactifications defined by arrangements. I. The ball quotient case, Duke Math. J. 118 (2003), no. 1, 151-187. MR1978885 (2004i:14042a) 5.1

[Mik06] G. Mikhalkin, Tropical geometry and its applications, ICM Lectures, Vol. II, Eur. Math. Soc., Zürich, 2006, pp. 827-852. MR2275625 (2008c:14077) 4

[OT92] P. Orlik and H. Terao, Arrangements of hyperplanes, Grundlehren der Mathematischen Wissenschaften, vol. 300, Springer-Verlag, Berlin, 1992. MR1217488 (94e:52014) 2.4

[Oxl11] J. Oxley, Matroid theory, second ed., Oxford Graduate Texts in Mathematics, vol. 21, Oxford University Press, Oxford, 2011. MR2849819 2.1, 2.2

[Pos09] A. Postnikov, Permutohedra, associahedra, and beyond, Int. Math. Res. Not. IMRN (2009), no. 6, 1026-1106. MR2487491 (2010g:05399) 5.5

[PS06] N. J. Proudfoot and D. Speyer, A broken circuit ring, Beiträge Algebra Geom. 47 (2006), no. 1, 161-166. MR2246531 (2007c:13029) 5.1, 5.1, 6.1

[RGST05] J. Richter-Gebert, B. Sturmfels, and T. Theobald, First steps in tropical geometry, Idempotent mathematics and mathematical physics, Contemp. Math., vol. 377, Amer. Math. Soc., Providence, RI (2005), pp. 289-317. MR2149011 (2006d:14073) 4

[Sch03] A. Schrijver, Combinatorial optimization. Polyhedra and efficiency. Vol. B, Algorithms and Combinatorics, vol. 24, Springer-Verlag, Berlin, 2003, Matroids, trees, stable sets, Chapters 39-69. MR1956925 (2004b:90004b) 2.5

[SSV11] R. Sanyal, B. Sturmfels, and C. Vinzant, The entropic discriminant, Adv. Math., to appear. arXiv:1108.2925 5.1, 5.1, 5.2

[ST09] H. Schenck and Ş O. Tohăneanu, The Orlik-Terao algebra and 2-formality, Math. Res. Lett. 16 (2009), no. 1, 171-182. MR2480571 (2010h:13023) 5.1, 5.1

[Stu96] B. Sturmfels, Gröbner bases and convex polytopes, University Lecture Series, vol. 8, American Mathematical Society, Providence, RI, 1996. MR1363949 (97b:13034) 4.1

[Stu02] B. Sturmfels, Solving systems of polynomial equations, CBMS Regional Conference Series in Mathematics, vol. 97, Published for the Conference Board of the Mathematical Sciences, Washington, DC, 2002. MR1925796 (2003i:13037) 4.1, 4.3, 4.6

[Suc13] A. I. Suciu, Hyperplane arrangements and Milnor fibrations, this volume, 2013. 2.14 
[Tev07] J. Tevelev, Compactifications of subvarieties of tori, Amer. J. Math. 129 (2007), no. 4, 1087-1104. MR2343384 (2008f:14068) 5.2, 5.2,6

[Var11] A. Varchenko, Quantum integrable model of an arrangement of hyperplanes, SIGMA Symmetry Integrability Geom. Methods Appl. 7 (2011), Paper 032, 55. MR2804564 (2012f:82034) 2.14

[Whi77] N. L. White, The basis monomial ring of a matroid, Advances in Math. 24 (1977), no. 3, $292-297$. MR0437366 (55 \#10297) 3.2

[Zie95] G. M. Ziegler, Lectures on polytopes, Graduate Texts in Mathematics, vol. 152, Springer-Verlag, New York, 1995. MR1311028 (96a:52011) 2.3

G. Denham, Department of Mathematics, University of Western Ontario, London, On N6A 5B7, CANADA

E-mail address: gdenham@uwo.ca 